\title{
Xueshuantong Improves Functions of Lymphatic Ducts and Modulates Inflammatory Responses in Alzheimer's Disease Mice
}

\author{
Rui Zheng, Yang-mei Huang and Qiang Zhou* \\ State Key Laboratory of Chemical Oncogenomics, Guangdong Provincial Key Laboratory of Chemical Genomics, Peking \\ University Shenzhen Graduate School, Shenzhen, China
}

Recent studies have revealed significant contributions of lymphatic vessels (LVs) to vital functions of the brain, especially related to clearance of waste from the brain and immune responses in the brain. These studies collectively indicate that enhancing the functions of LVs may improve brain functions during brain aging and in Alzheimer's disease (AD) where LV functions are impaired. However, it is currently unknown whether this enhancement can

OPEN ACCESS

Edited by: Luca Rastrelli,

University of Salerno, Italy

Reviewed by:

Ruxiang Xu,

Army General Hospital of People

Liberation Army, China

Thomas Van Groen,

University of Alabama at Birmingham,

United States

*Correspondence:

Qiang Zhou

zhouqiang@pkusz.edu.cn

Specialty section: This article was submitted to

Ethnopharmacology,

a section of the journal

Frontiers in Pharmacology

Received: 15 September 2020 Accepted: 01 September 2021 Published: 28 September 2021

Citation: Zheng $R$,

Huang Y-m and Zhou Q (2021) Xueshuantong Improves Functions of

Lymphatic Ducts and Modulates Inflammatory Responses in

Alzheimer's Disease Mice.

Front. Pharmacol. 12:605814. doi: 10.3389/fphar.2021.605814 be achieved using small molecules. We have previously shown that a widely used Chinese herbal medicine Xueshuantong (XST) significantly improves functions and reduces pathology in $A D$ transgenic mice associated with elevated cerebral blood flow (CBF). Here, we show that XST partially rescues deficits in lymphatic structures, improves clearance of amyloid- $\beta$ ( $A \beta$ ) from the brain, and reduces the inflammatory responses in the serum and brains of transgenic AD mice. In addition, we showed that this improvement in the lymphatic system occurs independently of elevated CBF, suggesting independent modulation and limited interaction between blood circulation and lymphatic systems. Moreover, XST treatment leads to a significant increase in GLT-1 level and a significantly lower level of MMP-9 and restores AQP4 polarity in APP/PS1 mice. These results provide the basis for further exploration of XST to enhance or restore LV functions, which may be beneficial to treat neurodegenerative diseases or promote healthy aging.

Keywords: Alzheimer's disease, lymphatic vessels, XST, ceftriaxone, APP/PS1 mice

\section{INTRODUCTION}

New drugs are urgently needed for Alzheimer's disease (AD), especially those that do not target amyloid- $\beta(A \beta)$ and tau since a variety of those drugs have shown minimal effectiveness in the clinical trials (Polanco et al., 2018; Zhu et al., 2019). Reduced cerebral blood flow (CBF) is reported in both $\mathrm{AD}$ patients and mice and has been linked to various pathologies in AD (Kisler et al., 2017; Kametani and Hasegawa, 2018; Vogelsang et al., 2018). Limited evidence suggests that improving CBF leads to improved functions and reduced pathology in AD transgenic mice (Kisler et al., 2017; Bracko et al., 2019). Recent studies also highlight the important contributions of lymphatic vessels (LVs) to the impaired brain functions in aging and in $\mathrm{AD}$ (van der Kleij et al., 2018; Da Mesquita et al., 2018a; Da Mesquita et al., 2018b). Treating aged mice with vascular endothelial growth factor C (VEGF-C) enhances meningeal lymphatic drainage of macromolecules from the cerebrospinal fluid and improves brain perfusion and learning/memory functions (Karaman et al., 2018; Patel et al., 
2019). Therefore, the ability to correct or compensate for the deficits in LV structure and functions and restore the brain clearance system is of great interest and importance for promoting healthy aging and treating neurodegenerative diseases (such as AD). No small molecule compound is known to modulate the structure/function of LVs. Given the importance of the lymphatic system, such small molecules may have significant therapeutic potentials.

LVs run within dural leaflets, on the inside surface of the dura, or in the subarachnoid space with the cortical veins (Thomas et al., 2019). Extensive meningeal LV network serves to clear macromolecules and regulate immune cell trafficking in the brain (Tarasoff-Conway et al., 2016; Bálint et al., 2019). This LV system drains interstitial fluids containing wastes from tissues and supports immune surveillance of the brain via recirculating immune cells (Venero Galanternik et al., 2016). In the lymphatic network, fluid, cells, and macromolecules are first absorbed by blind-ended lymphatic capillaries (initial LVs) (Klotz et al., 2015; Dave et al., 2018), proceed via collector LVs and lymph nodes, and end in the thoracic duct and right lymphatic trunk where lymphatic fluids are delivered into subclavian veins in the neck region (Aspelund et al., 2016; Bálint et al., 2019). Cerebrospinal fluid (CSF) enters the venous system via arachnoid granulations (Kortela et al., 2017), while macromolecules and immune cells in the CSF are transported along the dural LVs into the lymph nodes and extracranial systemic circulation and may induce immune responses (Shechter et al., 2013; Schläger et al., 2016). But how exactly blood circulation and lymphatic functions may interact with each other is poorly understood.

Function of LVs is compromised during aging and in neurodegenerative diseases, such as AD (Mesquita et al., 2018; Da Mesquita et al., 2018b; Sun et al., 2018b). Impaired meningeal lymphatic function slows down the perivascular influx of macromolecules into the brain and efflux of macromolecules from the interstitial fluid and induces cognitive impairment in mice (Guimaraes-Souza et al., 2019; Patel et al., 2019). Thus, LVs play an essential role in regulating homeostasis in the brain by draining macromolecules from cerebrospinal fluid (CSF) and interstitial fluid (ISF) into the cervical lymph nodes (CLNs) (Brouillard et al., 2014). Accumulation of $A \beta$ inside LVs of meninges of $A D$ patients or $A D$ transgenic mouse brains may contribute to $\mathrm{AD}$ progression ( $\mathrm{Da}$ Mesquita et al., 2018b; Sun et al., 2018b). Disruption of LVs in transgenic $\mathrm{AD}$ mice promotes $\mathrm{A} \beta$ deposition in the meninges which resembles human meningeal pathology, and this disruption aggravates parenchymal A $\beta$ accumulation (Kyrtsos and Baras, 2014; Mesquita et al., 2018).

In our previous study, we showed that a widely used Chinese herbal medicine Xueshuantong (XST) significantly elevates CBF, improves memory functions, and reduces $\mathrm{AD}$-relevant pathology in AD transgenic mice (APP/PS1) (Huang et al., 2018). Injected XST (lyophilized) (Supplementary Table S1) is a standardized product extracted from the rhizome comminution of Panax notoginseng [Araliaceae; Panax notoginseng (Burkill) F.H.Chen] and has been approved in treating stroke by the State Food and Drug Administration in China since 2002 (Wang et al., 2018). XST contains Notoginsenoside R1 (11.1\%), Ginsenoside Rg1 (48.1\%), Ginsenoside Re (5.5\%), Ginsenoside Rb1 (27.8\%), and Ginsenoside Rd (1.3\%)
(Supplementary Figure S1) (Yao et al., 2017; Wang et al., 2018). It is reported that XST significantly reduces inflammatory responses through NRF-2/Keap1 Pathway (Du et al., 2015; Wang et al., 2018), but whether this effect is mediated by elevated CBF is not known. In this study, we examined whether the same XST treatment as we used previously alters LV structure in APP/PS1 mice, and if so, whether such changes are associated with improved brain clearance of $\mathrm{A} \beta$ from the brain parenchyma. We also examined whether XST affects inflammatory responses in AD mice. Moreover, we examined whether enhanced CBF affects LVs in order to understand potential interactions between blood circulation and lymphatic system.

\section{MATERIALS AND METHODS}

Animals. APPswe/PSEN1dE9 mice with a C57BL/6 background were obtained from the Jackson Laboratory. APP/PS1 mice and wild-type littermate mice were genotyped by PCR analysis of genomic DNA. All experiments have been approved by the Peking University Shenzhen Graduate School Animal Care and Use Committee (Permit Number: AP0011) and were in accordance with the ARRIVE guidelines on the Care and Use of Experimental Animals. Male and female animals were fed separately and housed in a group of 4-5. The total number of APP/PS1 mice is 72 and wild-type mice is 32 . Males and females were used equally in all studies. We also found no impact of sex on the pathology of lymphatic drainage at baseline (Supplementary Figure S4), consistent with previous studies (Da Mesquita et al., 2018b). All mice were maintained under standard laboratory conditions at $22 \pm 2^{\circ} \mathrm{C}$, with $50 \pm 10 \%$ relative humidity, and on a $12 \mathrm{~h}$ light/dark cycle, with food and water available ad libitum. All mice were 11 months old.

Drugs. Xueshuantong (XST) (batch number: Z45021769) was obtained from Wuzhou Pharmaceutical Co., Ltd., China. The manufacturing technology was based on "Pharmacopoeia of China 2005." The rhizome comminution of Panax notoginseng [Araliaceae; Panax notoginseng (Burkill) F.H.Chen] was extracted by $60 \%$ ethanol under reflux three times ( $3 \mathrm{~h}$ per time). The extracting solution was merged for decompressing concentration till there was no alcohol taste and it was further subjected to a D101 macroporous absorption resin column eluted with water, $80 \%$ ethanol. The $80 \%$ ethanol extract was dried under vacuum to obtain XST (Wang et al., 2015). Previous studies have fully studied the main compositions and contents of this preparation (Yao et al., 2017; Zhang et al., 2020). The HPLC fingerprint shows that it contains Notoginsenoside R1 $(11.1 \%$, PubChem CID: 441934), Ginsenoside Rg1 (48.1\%, PubChem CID: 441923), Ginsenoside Re (5.5\%, PubChem CID: 441921), Ginsenoside Rb1 (27.8\%, PubChem CID: 9898279), and Ginsenoside Rd (1.3\%, PubChem CID: 11679800) (Yao et al., 2017). Freeze-dried powder of XST was dissolved in saline and administrated intraperitoneally (100 mg/kg), and saline injection was used as control. Dose and route of Xueshuantong administration used in the present study were based on a commonly used dosage of Xueshuantong in clinical practice 
and previous preclinical studies (Guo et al., 2018; Huang et al., 2018; Li et al., 2020). For experiments with 30-day injection (i.p.), 15 daily injections with a break of days were used. For experiments with 15-day injection (i.p.), daily injection was used. For examining short-term (5 d) effects, one injection was used.

Ceftriaxone (Cef) (Sigma) was dissolved in saline and administrated intraperitoneally $(20 \mathrm{mg} / \mathrm{kg})$, and saline injection was used as control. For experiments with 15-day injection (i.p.), daily injections were used. All tests were performed $24 \mathrm{~h}$ after the end of the last treatment.

Meninge/Tissue Collection and Processing. Mice were anesthetized with pentobarbital sodium $(1 \%, 30 \mathrm{mg} / \mathrm{kg}$, i.p.) and perfused with $0.1 \mathrm{M}$ PBS. After removing mandibles and skull rostral to the maxillae, the top of the skull was removed with surgical scissors. Whole-mount meninges were fixed while still attached to the skull cap using $4 \%$ paraformaldehyde (PFA) for $24 \mathrm{~h}$ at $4^{\circ} \mathrm{C}$. Fixed meninges (dura mater and arachnoid) were carefully dissected from the skullcaps with \#5 forceps (Fine Science Tools) and kept in PBS at $4^{\circ} \mathrm{C}$ until further use. Fixed brains were then washed with PBS, cryoprotected with $30 \%$ sucrose, and frozen in Tissue-Plus OCT compound (Thermo Fisher Scientific). Fixed and frozen brain sections $(30 \mu \mathrm{m}$ thickness) were cut on a cryostat (Leica) and kept in PBS at $4^{\circ} \mathrm{C}$. Frozen lymph nodes were sliced to $30 \mu \mathrm{m}$ thickness and collected onto gelatin-coated Superfrost Plus slides (Thermo Fisher Scientific) and stored at $-20^{\circ} \mathrm{C}$.

Immunohistochemistry. Tissue was rinsed in PBS and washed with PBS and $0.5 \%$ Triton X-100 for $10 \mathrm{~min}$, followed by incubation in PBS and $0.5 \%$ Triton X-100 containing $0.5 \%$ serum (goat or chicken) and $0.5 \%$ bovine serum albumin (BSA) for $1 \mathrm{~h}$ at room temperature. Then sections were incubated with primary antibodies: anti-CD31 (eBioscience, clone 390, 1:100), anti-Lyve-1 (eBioscience, clone ALY7, 1:200), anti-CD3e (eBioscience, clone 17A2, 1:500), anti-A $\beta$ (Abcam, ab10148, 1:500), anti-GFAP (Abcam, ab4674, 1: 500), and anti-Iba1 (Abcam, ab178847, 1:500), overnight at $4^{\circ} \mathrm{C}$ in PBS containing $1 \%$ BSA and $0.5 \%$ Triton X-100. Whole mounts and sections were then washed three times $(5 \mathrm{~min}$ each) at room temperature in PBS, followed by incubation with Alexa Fluor 488/594/647 chicken/goat anti-rabbit/goat IgG antibodies (Invitrogen, 1:1,000) for $1 \mathrm{~h}$ at room temperature in PBS with $1 \%$ BSA and $0.5 \%$ Triton X-100. After $5 \mathrm{~min}$ in DAPI (Invitrogen, 1 : 10,000 ), whole-mount sections were washed with PBS and mounted with Aqua-Mount (Lerner). Preparations were stored at $4^{\circ} \mathrm{C}$ for no more than 1 week before images were acquired on a Nikon AR1 confocal system (Nikon Corporation).

Enzyme-Linked Immunosorbent Assay (ELISA). ELISAs were performed to detect IL-10, IL-6, and IL-1 $\beta$ using specific kits (Boshide Bio, China). All blood samples of APP/PS1 or C57 mice, control, or XST-treated group were harvested and cryopreserved at $-80^{\circ} \mathrm{C}$ until analysis. All kits were used according to the manufacturer's suggested protocol.

Western Blot. As our previously described (Gao et al., 2019), tissues were collected, homogenized in RIPA buffer (Beyotime Biotechnology, China) containing $1 \mathrm{mM}$ PMSF (Sigma), $25 \mu \mathrm{M}$ leupeptin (Sigma), and $1 \mu \mathrm{g} / \mathrm{ml}$ aprotinin (Sigma), and centrifuged at $4^{\circ} \mathrm{C}$ for $0.5 \mathrm{~h}$ at $10,000 \mathrm{~g}$, with the supernatant collected. Total protein lysates made from hippocampus or entire brain were mixed with SDS gel-loading buffer and heated for $5 \mathrm{~min}$ at $100^{\circ} \mathrm{C}$. Samples $(15 \mu \mathrm{g}$ protein in each group) were separated on $10 \%$ SDS-PAGE gels (Invitrogen, Carlsbad, CA, United States) and transferred to polyvinylidene difluoride membranes (Millipore, Bedford, MA, United States). Membranes were blocked for $1 \mathrm{~h}$ at room temperature with $5 \%$ nonfat milk in TBST (TBS containing $0.05 \%$ Tween 20) and then probed with a specific primary antibody (anti-AQP4, 1:1000, Cat. No. ab46182, Abcam, United Kingdom; anti-GLT1, 1:1000, Cat. No. ab41621, Abcam, United Kingdom; anti-MMP9, 1:500, Cat. No. ab38898, Abcam, United Kingdom; antiGAPDH, 1:10000, Cat. No. ab8245, Abcam, United Kingdom, anti- $\alpha$-Tubulin, 1:10000, Cat. No. 5335T, CST, United Kingdom) overnight at $4^{\circ} \mathrm{C}$. A horseradish peroxidase- (HRP-) conjugated secondary antibody (anti-mouse or anti-rabbit, NeoBioscience) was added for $2 \mathrm{~h}$ at room temperature. Immunopositive AQP4, GLT1, MMP-9, and GAPDH bands were scanned and densitometrically analyzed by automated ImageJ software (NIH Image, Version 1.61), and their total protein densities were expressed relative to GAPDH or $\alpha$-Tubulin signals.

Image Analysis. Images were acquired with a Nikon AR1 confocal system (Nikon Corporation) using the LAS AF software. All images were acquired with a $1,024 \times 1,024$ pixel resolution. Quantitative analysis was performed using FIJI software (NIH). Diameters of LVs and blood vessels (BVs) of the meninges were quantified using ImageJ. A standard length $(100 \mu \mathrm{m})$ was first marked on the images to be analyzed, then the structures to be analyzed were selected, and sizes of these identified structures were automatically measured by ImageJ. Percentage of T-cells was determined by counting the number of T-cells within the superior sagittal sinus (SSS) or transverse sinus (TS). T-cell density was calculated by dividing the number of $\mathrm{T}$ lymphocytes by the area of the field. The density of $A \beta$ was calculated by dividing the number of $A \beta$ plaques by the area of field.

AQP4 Expression Polarity Measurement. To calculate the AQP4 polarity for each image, each color channel was processed separately with each image thresholded uniformly at two different levels, a high- and a low-stringency threshold. The low-stringency threshold defined the overall area of AQP4-immunoreactivity while the highstringency threshold defined the area of intense AQP4immunoreactivity that in control mice is localized to perivascular endfeet. The ratio of low- and high-stringency area was used to generate an arbitrary value defined as "AQP4 polarity" (Supplementary Figure S3) (Wang et al., 2012). The higher the AQP4 polarity was, the greater the proportion of immunoreactivity was restricted to the perivascular regions, while the lower the proportion, the more the evenly distributed immunoreactivity was between the perivascular endfeet and the soma.

Two-Photon Time-Lapse Imaging of Blood Flow. As previously described (Huang et al., 2018), mice were injected with Cef or saline daily for 15 days, and microvessels were imaged on day 15 using two-photon imaging using Olympus BX 51 microscope. To measure blood flow in the microvessels (diameter 7-15 $\mu \mathrm{m}$ or $40-60 \mu \mathrm{m}$ ), $25 \mu \mathrm{l}$ of $10 \%$ fluorescein sodium salt (Sigma-Aldrich) was injected through the mouse tail vein $1 \mathrm{~h}$ prior to imaging. Several frame images with different zooms were collected to facilitate the identification of the same set of vessels by using 
A

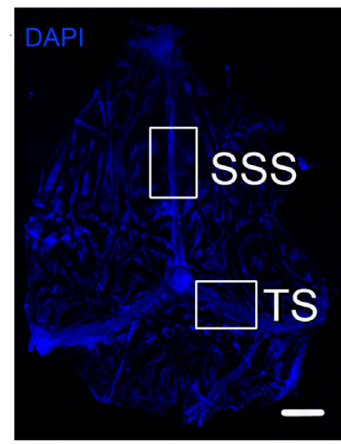

D

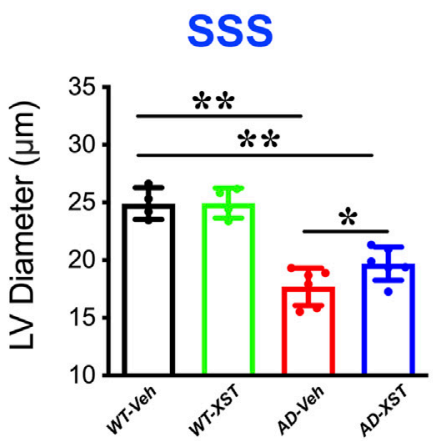

$\mathbf{F}$

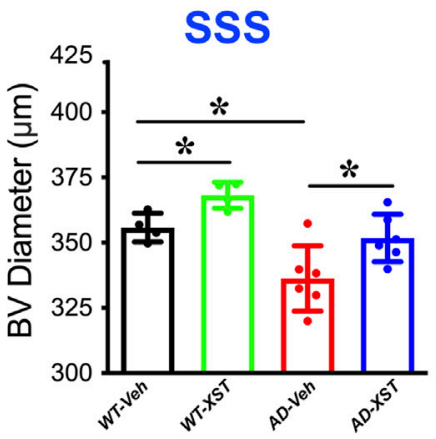

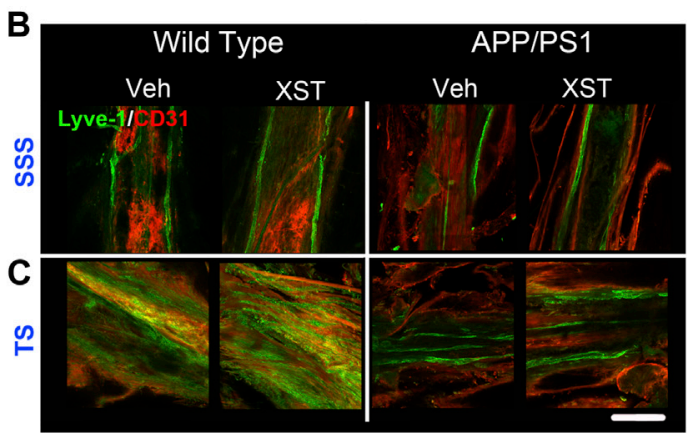

E

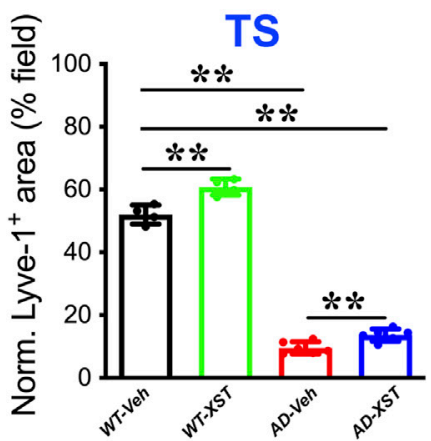

G

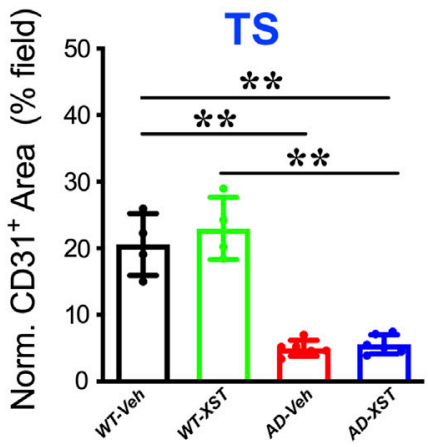

FIGURE 1 | Effects of XST on lymphatic structures. (A) Schematic representation of the whole-mount dissection of the dura mater. SSS, superior sagittal sinus; TS, transverse sinus. Scale bar, 2,000 $\mu \mathrm{m}$. (B) Sample images of lymphatic vessels (LVs) (Lyve-1) and blood vessels (BVs) (CD31) in the SSS region, in wild-type and APP/ PS1 mice treated with either XST or Vehicle (Veh). C57-Veh ( $n=4$ mice), C57-XST ( $n=4$ mice), AD-Veh $(n=6$ mice), AD-XST ( $n=6$ mice). Scale bar, 200 $\mu$ m. (C) Sample images of lymphatic ducts (Lyve-1) and BVs (CD31) in the TS region, in wild-type and APP/PS1 mice treated with either XST or Veh. Scale bar, 200 $\mu$ m. (D) Quantification of the diameter of lymphatic ducts in mice treated with XST or Veh in the SSS region $\left(F_{(1,16)}=87.18, C 57\right.$ vs. AD, $p<0.0001 ; A D-V e h$ vs. AD-XST, $p=$ 0.034, two-way ANOVA with Bonferroni's multiple comparisons). (E) Quantification of the area occupied by lymphatic ducts in mice treated with XST or Veh in the TS region $\left(F_{(1,16)}=36.92\right.$, C57 vs. AD, $p<0.0001$; C57-Veh vs. C57-XST, $p<0.001$; AD-Veh vs. AD-XST, $p=0.0454$, two-way ANOVA with Bonferroni's multiple comparisons). (F) Quantification of the diameter of BVs in mice treated with XST or Veh in the SSS region $\left(F_{(1,16)}=22.18, C 57\right.$ vs. AD, $p=0.0002 ; C 57-V e h$ vs. C57-XST, $p=0.049$; AD-Veh vs. AD-XST, $p=0.0303$, two-way ANOVA with Bonferroni's multiple comparisons). (G) Quantification of the area occupied by BVs in mice treated with XST or Veh in the TS region ( $F_{(1,16)}=141.4$, C57 vs. AD, $p<0.0001$; two-way ANOVA with Bonferroni's multiple comparisons). Data are mean \pm SEM. ${ }^{\star} p<0.05$; ${ }^{* \star} p<0.01$

landmarks in these images. The speed of the movement of the RBCs was calculated from the strips in the line scan images. The instantaneous velocity of RBCs is calculated as $\mathrm{V}=\Delta \mathrm{y} / \Delta \mathrm{t}$; flux = $1 / \Delta \mathrm{t}$; linear density $=1 / \Delta \mathrm{y}$.

Statistical Analysis. Data were analyzed using GraphPad Prism 8 software. Unpaired $t$-test, paired $t$-test, and two-way repeated measures ANOVA followed by Bonferroni posttest were used. All results were shown as mean \pm SEM. $p<0.05$ was considered statistically significant.

\section{RESULTS}

\section{Altered Structures of LVs in APP/PS1 Mice and Improvement by XST Treatment}

By using immunostaining, we imaged the structure of LVs in both APP/PS1 and WT mice, treated with XST or Vehicle (Veh). Two regions were examined, SSS and TS (Figure 1A), where LVs have been shown to be altered in both $\mathrm{AD}$ and during aging (Mesquita 

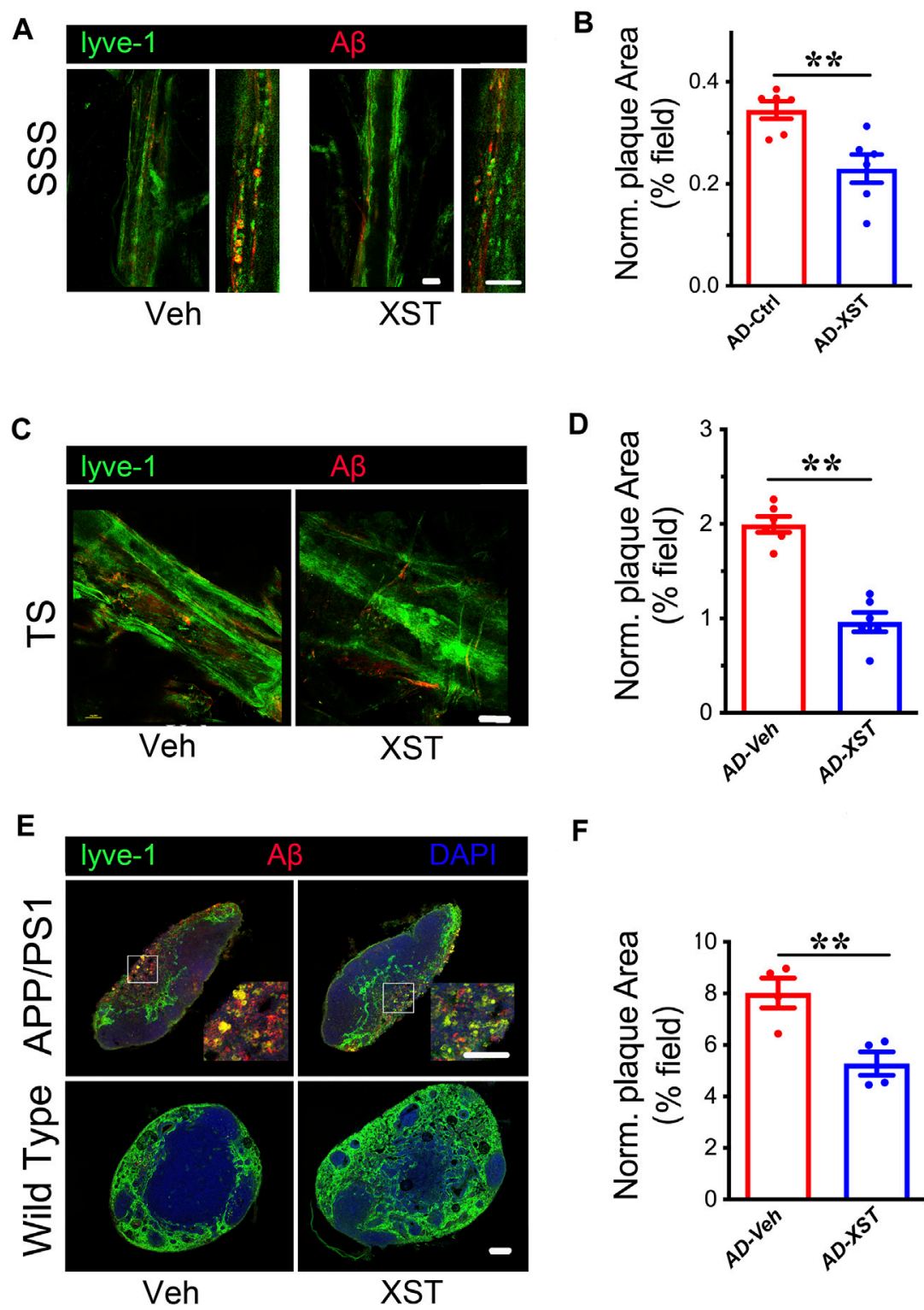

$\mathbf{F}$

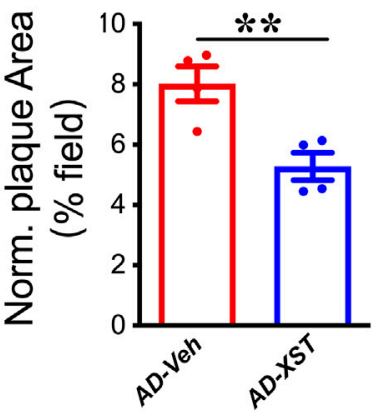

FIGURE 2 |XST improves A $\beta$ removal from brains of APP/PS1 mice. (A) Impact of XST on the removal of A $\beta$ via the brain lymphatic ducts in APP/PS1 mice, in the SSS and TS regions. Red, A $\beta$; green, Lyve-1. Scale bars, $100 \mu \mathrm{m}$. (B) Quantification of plaque area in the SSS region in XST- or Veh-treated group $(p=0.0056, t$-test, $n=$ 6 mice per group). (C) Impact of XST on A $\beta$ removal via the brain lymphatic ducts in APP/PS1 mice, in the TS regions. Red, A $\beta$; green, Lyve-1. Scale bar, 100 $\mu m$. (D) Quantification of plaque area in the TS region XST- or Veh-treated group $(p=0.0067, t$-test, $n=4$ mice per group). (E) Sample images if $A \beta$ staining in the lymph nodes of the neck. Red, A ; ; green, Lyve-1; blue, DAPI. Scale bar, $200 \mu \mathrm{m}$ (low). Scale bar, $100 \mu \mathrm{m}$ (high). (F) Quantification of plaque area in the lymph nodes of the neck region in XST- or Veh-treated APP/PS1 mice ( $p=0.0098, t$-test, $n=4$ mice per group). Data are mean \pm SEM. ${ }^{*} p<0.05 ;{ }^{*} p<0.01$.

et al., 2018). In the SSS region, diameters of LVs in APP/PS1 mice were significantly smaller than those in WT mice (Figure 1B; Lyve- $1^{+}$; Figure 1D; $\left.p<0.01\right)$, consistent with previous results on defected LVs in AD brains (Da Mesquita et al., 2018b). The XSTtreated APP/PS1 group, compared to the Veh-treated group, had significantly larger LV diameters, a change not observed in WT mice (Figure 1D; $p<0.05$ ). Since LV diameters cannot be reliably measured in the TS region, we measured areas positive for Lyve-1 in TS (\% of entire field) (Patel et al., 2019). We found a significant reduction in the Lyve- $1^{+}$area in the APP/PS1 mice compared to
WT mice (Figures 1C,E; $p<0.01$ ), while these areas were significantly larger in XST-treated mice (both APP/PS1 and WT) (Figures 1C,E; $p<0.01$ ).

In addition to LVs, we also measured BVs attached to the meninges. In the SSS region of both APP/PS1 and WT mice (Figure 1B; $\mathrm{CD} 31^{+}$), we found a significant reduction in the diameters of $\mathrm{CD} 31^{+} \mathrm{BVs}$ in APP/PS1 mice compared to WT mice (Figures 1B,F; $p<0.05$ ), while XST-treated group exhibited significantly larger diameters in both APP/PS1 and WT mice (Figures 1B,F; $p<0.05$ ). In the TS region, there was a significant 


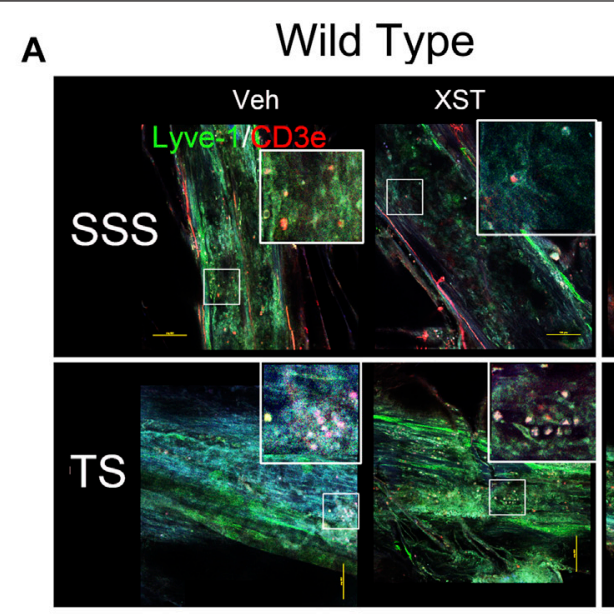

B

D
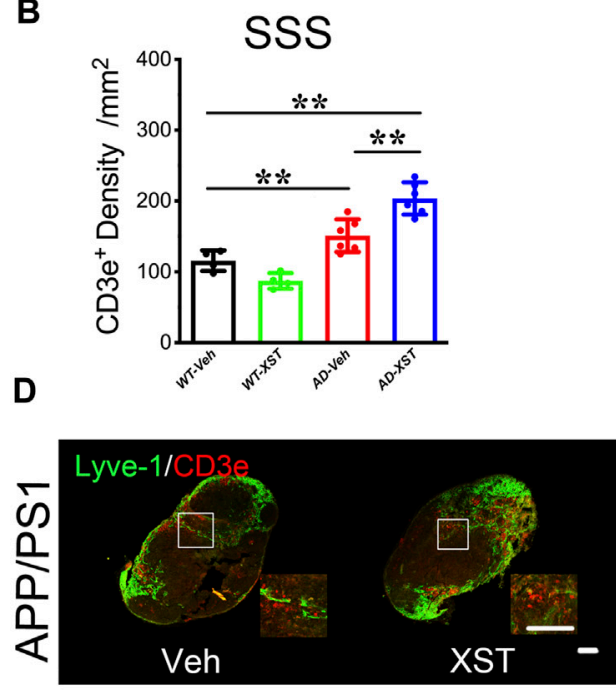

F

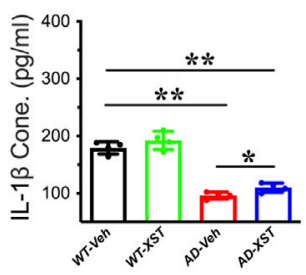

G

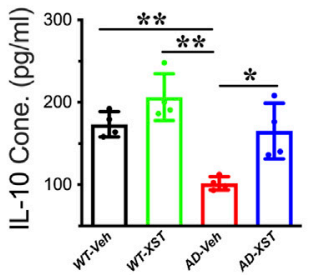

APP/PS1

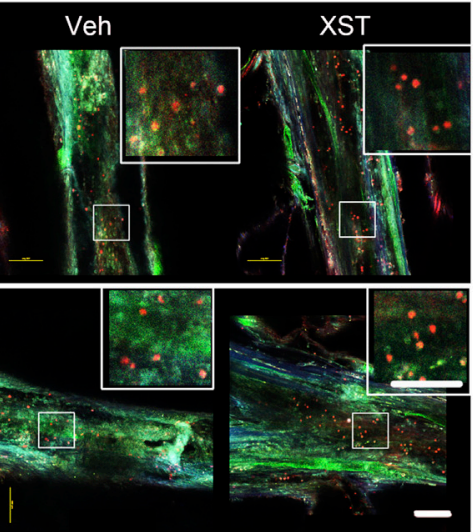

C
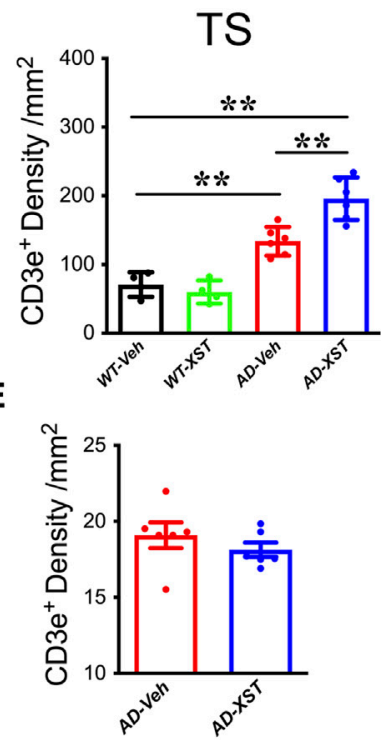

H

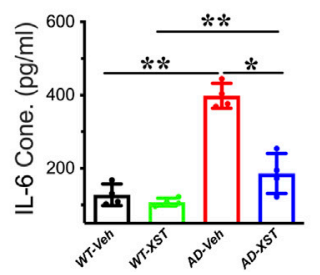

FIGURE 3 | Effect of XST on inflammatory responses in the brains of APP/PS1 mice. (A) Sample images of lymphatic ducts (Lyve-1, green) and T-cells (CD3e, red) in SSS region and TS region, in WT and APP/PS1 mice treated with either XST or Veh. Scale bar, $100 \mu \mathrm{m}$. (B) Quantification of T-cell density in lymphatic ducts of the SSS region in WT and APP/PS1 mice treated with XST or Veh $\left(F_{(1,16)}=70.96\right.$, C57 vs. AD, $p<0.0001$; AD-Veh vs. AD-XST, $p=0.0017$, two-way ANOVA with Bonferroni's multiple comparisons). (C) Quantification of the T-cell density in lymphatic ducts of the TS region in WT and APP/PS1 mice treated with XST or Veh $\left(F_{(1,16)}=86.28\right.$, C57 vs. AD, $p<0.0001 ; A D-V e h$ vs. AD-XST, $p=0.0019$, two-way ANOVA with Bonferroni's multiple comparisons). (D) Sample images of T-cells in the lymph nodes of the neck. T-Cells (CD3e, red); lymph nodes (Lyve-1, green). Scale bar, $200 \mu \mathrm{m}$ (low). Scale bar, $100 \mu \mathrm{m}$ (high). (E) Quantification of T-cells density in the lymph nodes of the neck region in XST- or Veh-treated APP/PS1 mice ( $p=0.3483$, $t$-test, $n=6$ mice per group). Quantitative measurement of IL-1 $\beta$ (F) $\left(F_{(1,12)}=232.5\right.$, C57 vs. AD, $p<0.0001$; AD-Veh vs. AD-XST, $p=0.0279$, two-way ANOVA with Bonferroni's multiple comparisons); IL-6 (G) $\left(F_{(1,12)}=94.89\right.$, C57 vs. AD, $p<0.0001$; AD-Veh vs. AD-XST, $p=0.014$, two-way ANOVA with Bonferroni's multiple comparisons); and IL-10 (H) $\left(F_{(1,12)}=22.63, C 57\right.$ vs. AD, $p=0.0005$; AD-Veh vs. AD-XST, $p=0.0155$, two-way ANOVA with Bonferroni's multiple comparisons). Levels in serum in WT and APP/PS1 mice ( $n=4$ mice per group). Data are mean \pm SEM. IL: interleukin. ${ }^{\star} p<0.05 ;{ }^{*} p<0.01$. 
reduction in the $\mathrm{CD} 31^{+}$area in APP/PS1 mice compared to WT (Figures 1C,G; $p<0.01$ ), but no difference was seen between XST- and Veh-treated groups in either WT or APP/PS1 mice. Thus, the impacts of XST on BVs are brain region-dependent.

\section{Reduced $A \beta$ Presence in LVs in XST-Treated APP/PS1 Mice}

Previous studies indicated enhanced clearance ability with enlarged LVs (Da Mesquita et al., 2018a; Sun et al., 2018b). To test whether this is the case with XST, we examined A $\beta$ clearance in the brain after XST or Veh treatment by staining A $\beta$ in brain sections containing either SSS or TS region. We found that, in the XST-treated group, $\mathrm{A} \beta$-positive areas are significantly smaller (Figures $2 \mathbf{A}, \mathbf{B} ; p<0.01$ ), suggesting a more effective removal of $A \beta$ from LVs with XST treatment. We also found a significantly smaller $A \beta$ plaque area in the TS region of XST-treated APP/PS1 mice (Figures 2C,D; $p<0.01$ ). Due to the absence of $A \beta$ in WT mouse brains, this analysis was not performed.

Macromolecules in CSF are transported mainly along the dural LVs into lymph nodes of the neck and extracranial systemic circulation (Kisler et al., 2017; Da Mesquita et al., $2018 b$ ). It was reported that $A \beta$ is drained from TS into the lymph nodes along the side of the neck (Louveau et al., 2017). We then examined $A \beta$ plaques in regions where LVs merge with BVs in the lymph node (Figure 2E). A significantly smaller $A \beta$ area was seen in the XST-treated compared to Veh-treated APP/PS1 mice (Figures 2E,F; $p<0.01$ ). All together, these results indicate a reduced $A \beta$ plaque size consistent with our previous findings (Huang et al., 2018) and suggest that XST enhances A $\beta$ clearance capacity in APP/PS1 mice.

\section{Reduced Inflammatory Responses With XST Treatment}

Another reported consequence of enhanced LV structure/ function is an improved inflammatory response shown as higher T-cell density in the SSS region (Kim and Song, 2017). To examine this, we first counted the number of $\mathrm{CD} 3 \mathrm{e}^{+} \mathrm{T}$-cells in the SSS and TS regions (Figure 3A). Density of CD3e $\mathrm{e}^{+}$cells inside LVs was significantly higher in the APP/PS1 mice compared to WT mice (Figures 3A-C; $p<0.01$ for both regions), suggesting elevated immune responses in APP/PS1 mice (Toly-Ndour et al., 2011; Zhu et al., 2019). A significantly higher density of CD3e ${ }^{+}$ cells was seen in APP/PS1 mice treated with XST, compared to the Veh group (Figure 3B; $p<0.01$ ), suggesting a further increase in T-cell density and likely enhanced responses after XST treatment. When the same measurement was repeated in the TS region, we also found a significantly higher density of CD $3 \mathrm{e}^{+}$ cells in APP/PS1 mice in the XST-treated group compared to the Veh group (Figures 3A,C; $p<0.01$ ). These results suggest that XST may promote immune surveillance. However, the CD $3 \mathrm{e}^{+}$cell density in neck lump node (CLNs) was not different between the XST- and Veh-treated groups in the APP/PS1 mice (Figures 3D,E), suggesting that T-cell storage in CLNs is not affected by XST.
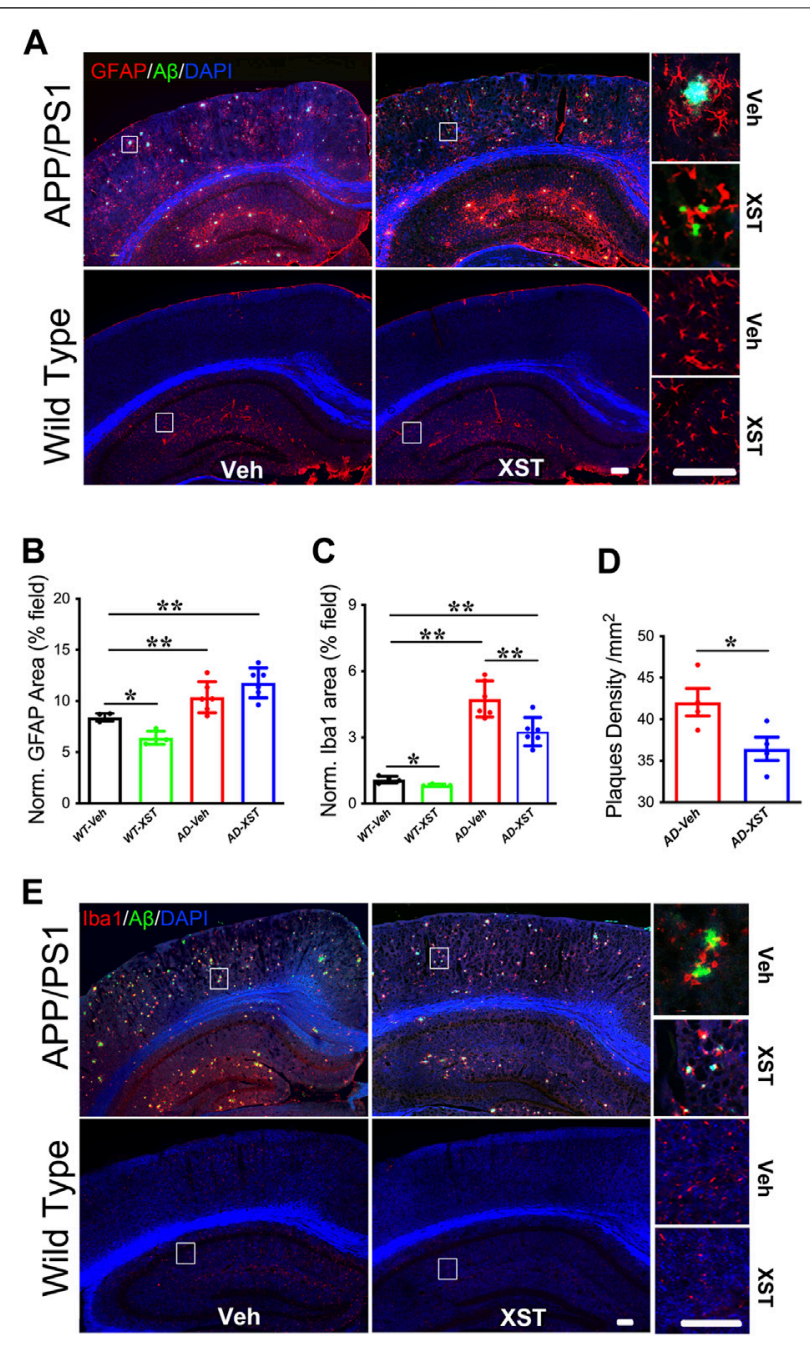

FIGURE 4 | Effect of XST on A $\beta$ removal from the brains of APP/PS1 mice. (A) Sample images of amyloid plaques (A $\beta$, green) and astrocytes (GFAP, red) in the cortex and hippocampus of WT or APP/PS1 mice. Scale bar, $100 \mu \mathrm{m}$. (B) Quantification of $\mathrm{GFAP}^{+}$area of in the brains of WT and APP/PS1 mice treated with XST or Veh $\left(F_{(1,16)}=43.53\right.$, C57 vs. AD, $p<$ 0.0001 ; C57-Veh vs. C57-XST, $p=0.038$, two-way ANOVA with Bonferroni's multiple comparisons). (C) Quantification of $A \beta$ density in the brains of APP/ PS1 mice treated with XST or Veh $\left(F_{(1,16)}=129.7\right.$, C57 vs. AD, $p<0.0001$; AD-Veh vs. AD-XST, $p=0.0028$, two-way ANOVA with Bonferroni's multiple comparisons). (D) Quantification of the area of $\mathrm{Iba1}^{+}$in the brain of WT and APP/PS1 mice treated with XST or Veh $(p=0.0409$, $t$-test, $n=4$ mice per group). (E) Sample images of amyloid plaque (A $\beta$, green) and microglia (lba1, red) in the cortex and hippocampus of WT or APP/PS1 mice. Scale bars,

$100 \mu \mathrm{m}$. Data are mean \pm SEM. ${ }^{*} p<0.05 ;{ }^{* *} p<0.01$.

The above results suggest an increased T-cell density which is expected to reduce inflammation in the brain. We next examined the serum level of IL-1 $\beta$ (a marker of anti-inflammatory response) (Williams et al., 1999). A significantly lower level of IL-1 $\beta$ was seen in APP/PS1 mice compared to WT mice (Figure 3F; $p<0.01$ ) and a significantly higher level in the XST-treated APP/PS1 mice (compared to Veh-treated) (Figure 3F; $p<0.05$ ). Consistently, serum IL-10 level was significantly higher in the XST-treated APP/PS1 mice than in 

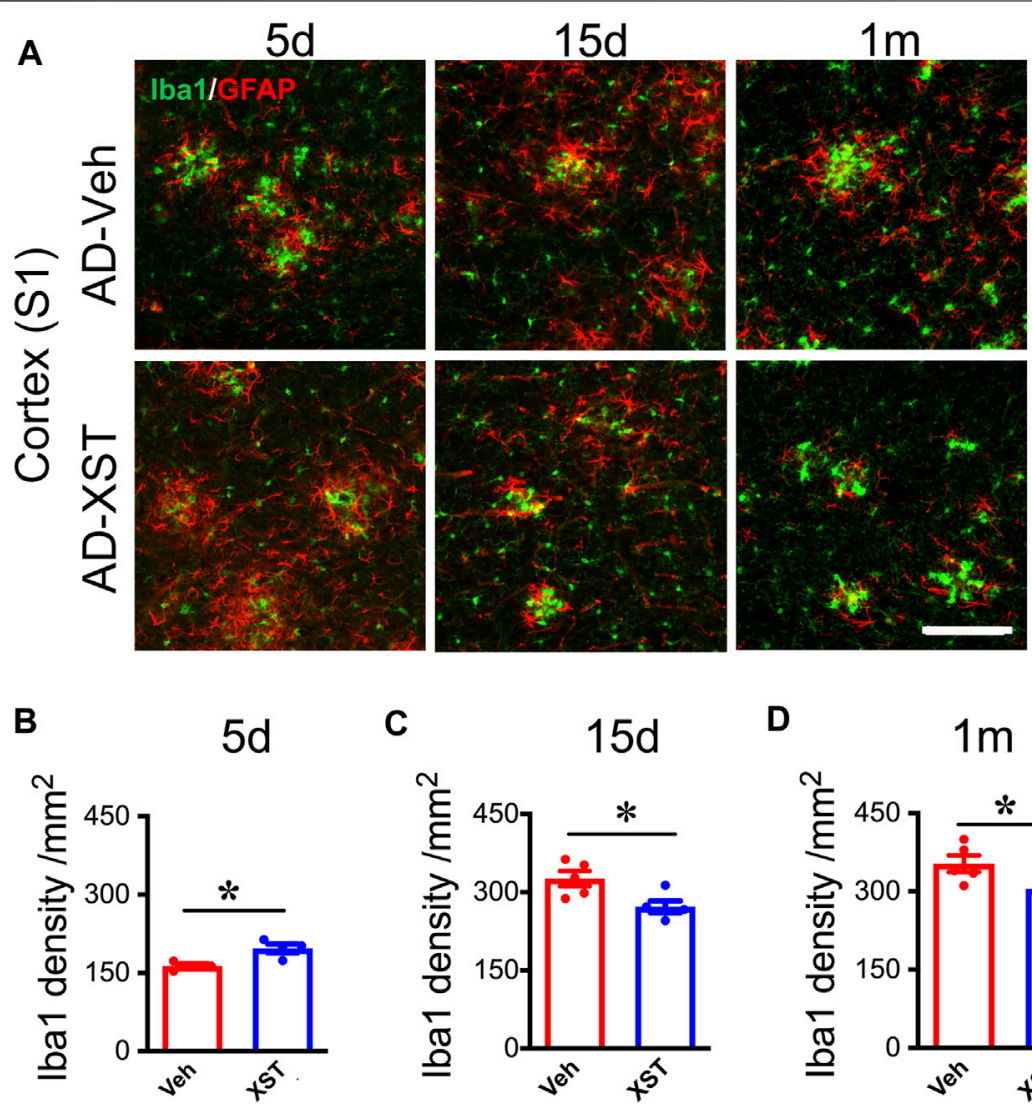

C

$15 d$

D

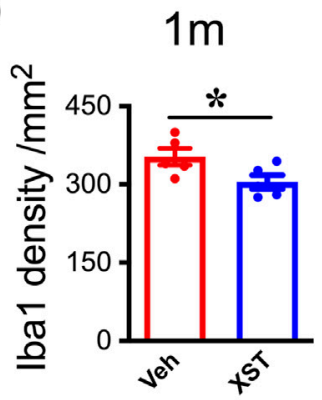

E

$5 d$
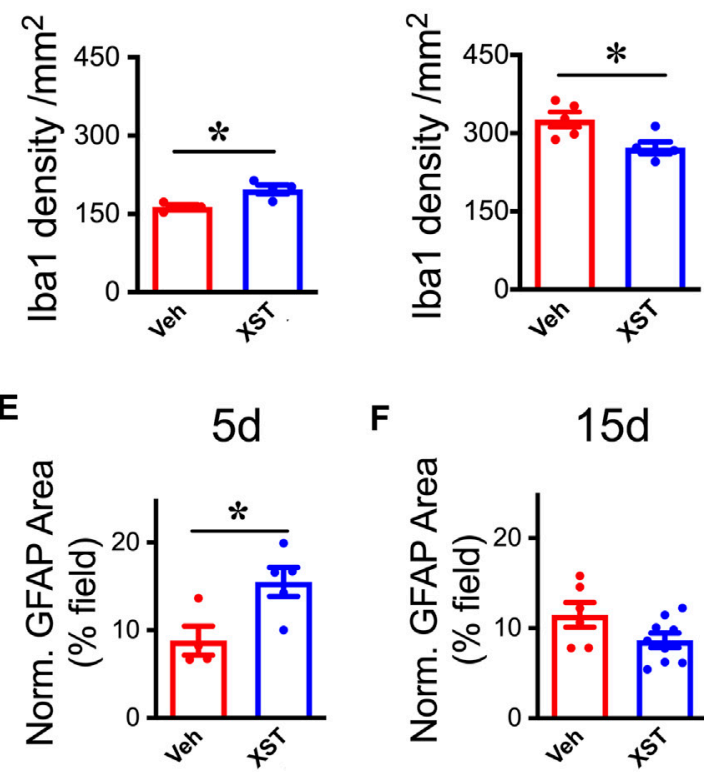

F

G
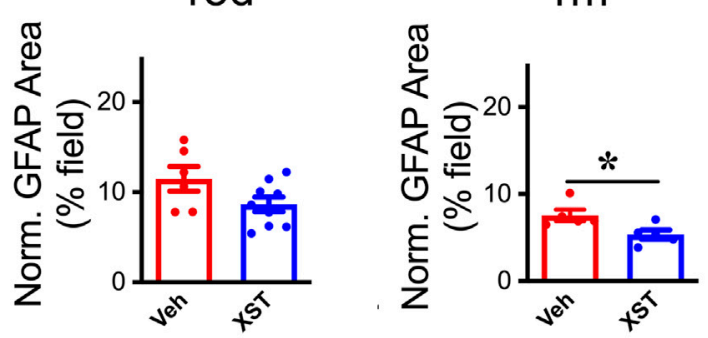

H

Hippocampus

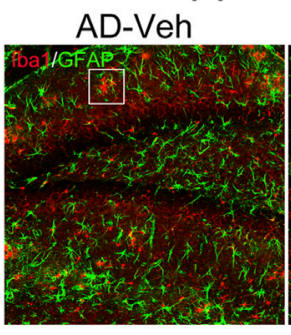

AD-XST
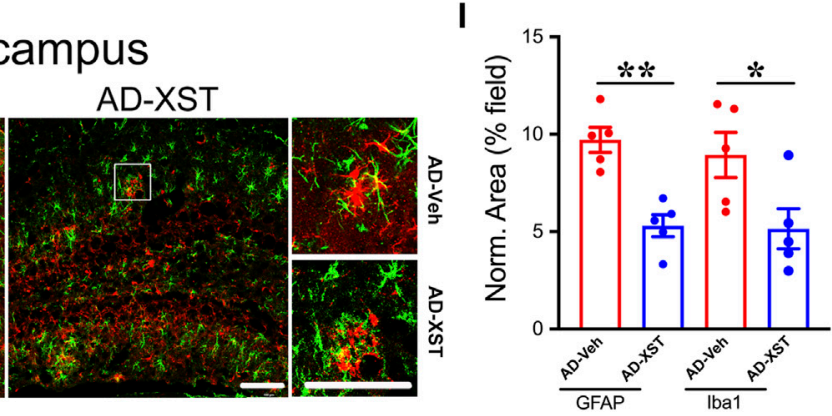

FIGURE 5 | Time course of XST's impact on microglia and astrocytes in APP/PS1 mice. (A) Sample images of microglia (Iba1, green) and astrocytes (GFAP, red) in the cortex (S1) of APP/PS1 mice after treatment with XST for 5 days, 15 days, or 1 month. Scale bar, $100 \mu \mathrm{m}$. (B-D) Quantification of Iba $1^{+}$density after 5 days (B) $(p=$ 0.0112, $t$-test, $n=4$ mice per group), 15 days (C) ( $p=0.0233$, $t$-test, AD-Veh, $n=5$ mice, AD-XST, $n=4$ mice), and 1 month (D) ( $p=0.0487, n=5$ mice per group) of XST treatment in APP/PS1 mice. (E-G) Quantification of GFAP ${ }^{+}$area after 5 days $\mathbf{( E )}(p=0.0253, t$-test, AD-Veh, $n=4$ mice, AD-XST, $n=5$ mice), 15 days $(\mathbf{F})(p=$ $0.0798, t$-test, AD-Veh, $n=6$ mice, AD-XST, $n=9$ mice), and 1 month (G) ( $p=0.0291, t$-test, $n=5$ per group) of XST treatment in APP/PS1 mice. (H) Sample images of microglia (Iba1, red) and astrocytes (GFAP, green) in the hippocampus of APP/PS1 mice after treatment with XST for 15 days. Scale bar, $100 \mu \mathrm{m}$. (I) Quantification of $\mathrm{GFAP}^{+}$area $\left(p=0.009\right.$, $t$-test, $n=5$ mice per group) and lba $1^{+}$area $(p=0.04, t$-test, $n=5$ mice per group) in APP/PS1 mice after treatment with XST for 15 days. Data are mean \pm SEM. ${ }^{*} p<0.05 ;{ }^{* *} p<0.01$. 
Veh-treated APP/PS1 mice (Figure 3G; $p<0.05$ ). On the other hand, serum IL-6 level (a proinflammation cytokine) was significantly higher in the APP/PS1 mice compared to WT mice (Figure 3H; $p<0.01$ ) and significantly lower in the XST-treated APP/PS1 mice compared to Veh group (Figure 3H; $p<0.05)$. In addition, we also found a significantly lower IL-6 level in the brain in the XST-treated APP/PS1 mice (Supplementary Figure S2C; $p<0.05$ ) and a significantly higher IL-10 level in the XST-treated APP/PS1 mice than in Veh-treated APP/PS1 mice (Supplementary Figure S2D; $p<0.01)$. Thus, these results suggest that the inflammation level is significantly reduced by XST in APP/PS1 mice.

\section{Cellular Markers of Inflammation in the Brain With XST Treatment}

Enhanced responsiveness of both microglia and astrocytes has been widely reported in both $\mathrm{AD}$ patients and $\mathrm{AD}$ mice (Fakhoury, 2018; Kaur et al., 2019). In the APP/PS1 mice, a larger area occupied by astrocytes $\left(\mathrm{GFAP}^{+}\right)$was found in APP/ PS1 mice (compared to WT mice) (Figures 4A,B; $p<0.01$ ), and $\mathrm{GFAP}^{+}$area was not different between XST- and Veh-treated groups (Figures 4A,B). Interestingly, XST-treated WT mice showed a small but significantly smaller $\mathrm{GFAP}^{+}$area (Figures 4A,B; $p<0.05)$. In the same mice, the areas covered by $\mathrm{Iba}^{+}$ cells were significantly larger in APP/PS1 mice (Figures 4C,E; $p<0.01)$ and significantly smaller in APP/PS1 and WT mice treated with XST [Figures 4C,E; $p<0.01$ (APP/PS1), $p<0.05$ (wt)]. In addition, we found a significantly smaller $A \beta$ plaque density in XST-treated APP/PS1 mice (Figures 4A,D; $p<0.05$ ), consistent with a reduced $A \beta$ level in the previous experiments (Figure 2). Together with the results in Figure 3, XST reduces the activation of astrocytes and microglia which may underlie the altered serum cytokine levels and enhanced clearance of $A \beta$ plaque.

\section{Time Course of Changes in Astrocytes and Microglia Density After XST Treatment}

In the above analysis, we conducted experiments at one-time point (15 days after XST treatment). To understand the time course of changes in astrocytes and microglia, we analyzed staining of GFAP and Iba1 at 5 days, 15 days, and 1 month in APP/PS1 mouse brains treated with either XST or Veh (Figure 5A). Interestingly, we found a significantly higher density of Ibal and bigger GFAP-positive area in mice treated with XST for 5 days (Figures 5A,B for Ibal; $p<0.05$; Figures 5A,E for GFAP; $p<0.05$ ). In mice treated with XST for 15 days and 1 month, we found a significantly lower density of Ibal (Figures 5A,C,D, $p<0.05$ ) and the trend towards reduced GFAPpositive area (Figures $\mathbf{5 A}, \mathbf{F}, \mathbf{G})$ in the cortex (S1). In addition, we also found significantly smaller GFAP-positive and Iba1-positive areas in the hippocampus of mice treated with XST for 15 days (Figures 5H,I, $p<0.01, p<0.05$ ). Thus, XST treatment leads to an initial increase in the density of both astrocytes and microglia, which is followed by a decrease in density.

\section{Elevating CBF Does Not Modulate LVs in APP/PS1 Mice}

The meningeal lymphatic system is necessary for effective clearance of macromolecules from the brain ISF and may serve as a common pathway for effective removal of wastes from brain parenchyma (Plog and Nedergaard, 2018; Chen et al., 2020). We have shown that XST elevated CBF in both APP/PS1 and WT mice (Huang et al., 2018), and thus elevated CBF could drive the improved LV structure/function. Since ceftriaxone (Cef) injection reliably increases CBF (Kortela et al., 2017; Holschneider et al., 2020), we first confirmed this in the APP/PS1 mice using two-photon linescan imaging (Huang et al., 2018). We have focused on small BVs (diameters 7-15 $\mu \mathrm{m}$ ) due to their reported alteration in $\mathrm{AD}$ (Ahn et al., 2018; Bracko et al., 2019) and used medium-sized vessels as control (diameters $40-60 \mu \mathrm{m})$. The velocity of blood flow in small BVs was significantly higher in the Cef-injected APP/PS1 mice compared to Veh-injected (Figure 6A, $p<0.01$ ), while no difference was seen in mid-size BVs between these two groups (Figure 6B). We then imaged the structures of LVs and BVs in APP/PS1 mice treated with Cef or Veh. Diameters of LVs in the SSS or TS region were not different between Cef- and Vehinjected groups (Figures 6C,D), while the diameters of BVs in the SSS region were also not different between Cef- and Veh-injected group (Figure 6E). Thus, elevating CBF by Cef injection is not sufficient to alter LV structure in APP/PS1 mice.

To understand whether $A \beta$ plaques could be affected by Cef treatment, we measured their density in the primary somatosensory cortex and found no difference between Cefand Veh-injected APP/PS1 mice (Figures 6F,G), suggesting that elevating $\mathrm{CBF}$ by Cef is not sufficient to modulate plaques, in contract to the significant reduction after XST treatment. Since significant alterations in astrocytes and microglia were seen in APP/PS1 mice after XST treatment (Figure 5), we measured the density of these cells in Ceftreated APP/PS1 mice. Microglia density (Iba1) was significantly lower in APP/PS1 mice treated with Cef for either 5 days or 15 days (Figures $\mathbf{6 H}, \mathbf{I}, p<0.05$ ), but the area of astrocyte (GFAP) was lower in the 15-day but not 5-day treatment group (Figures $\mathbf{6 H}, \mathbf{J}$ ). Thus, compared to XST, Cef treatment does not increase astrocytes and microglia density and thus may have a distinct impact on inflammatory responses. Taken together, although elevated CBF is seen after either XST or Cef in APP/PS1 mice, changes in LVs and A $\beta$ plaques were only seen after XST treatment, suggesting that elevated CBF is unlikely to underlie these differential changes in APP/PS1 mice with XST.

\section{Differential Changes in Protein Levels Induced by XST and Cef Treatment}

To further understand what could drive or underlie the observed distinct changes associated with XST or Cef treatment, we examined the levels of aquaporin 4 (AQP4) and glial glutamate transporter 1 (GLT-1) since they play important roles in regulating the exchange between brain parenchyma and CSF (Sharma et al., 2019; Simone et al., 2019). AQP4 
A

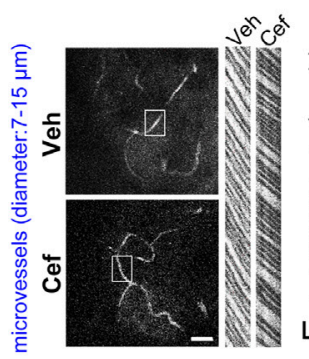

C

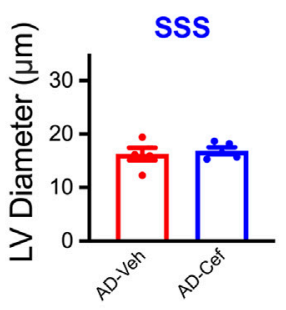

F

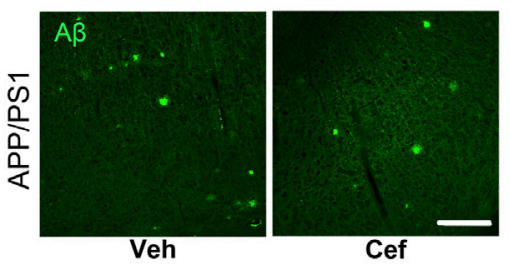

G

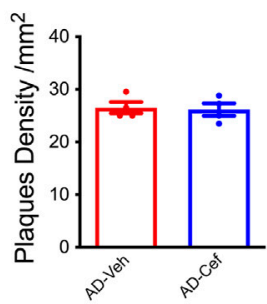

I

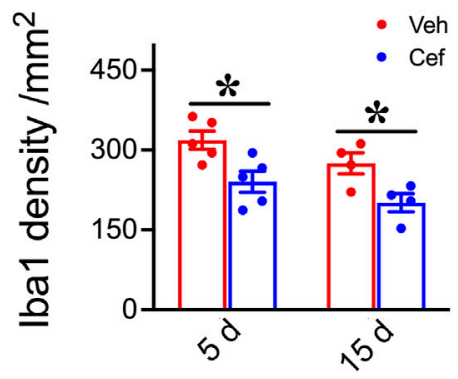

B

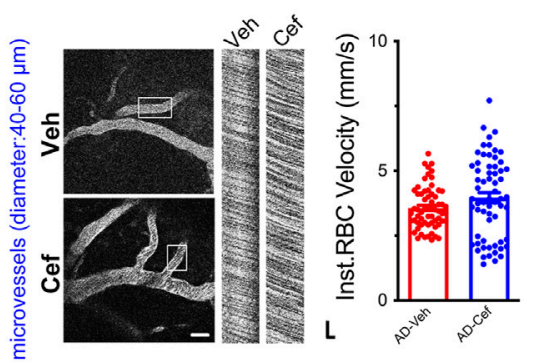

D

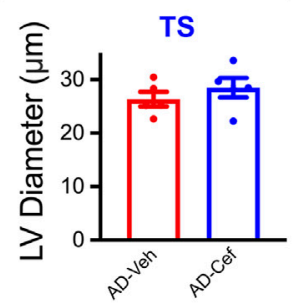

E

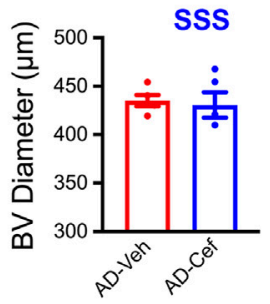

H
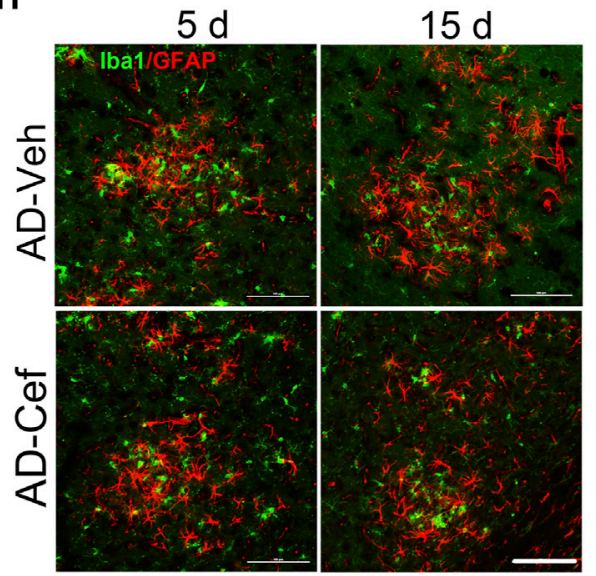

J

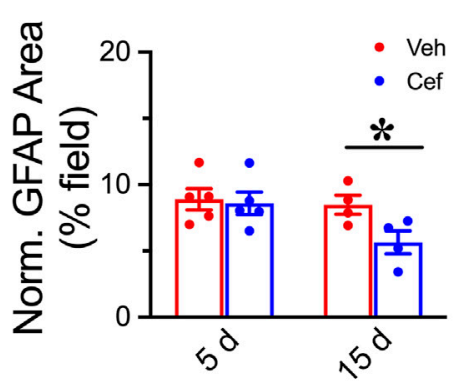

FIGURE 6 | Impacts of Cef on CBF and lymphatic vessel structure in APP/PS1 mice. (A) Sample image of small BVs (diameter, 7-15 $\mu$ m, left) and line scan image of blood flow (right). Scale bar, $100 \mu \mathrm{m}$ (left), $10 \mathrm{~ms}$, and $10 \mu \mathrm{m}$ (right) $(p=0.0003, t$-test, $n=64 \mathrm{BVs} / 4$ mice per group). (B) Sample image of large BVs (diameter, 40-60 $\mu \mathrm{m}$, left) and line scan image of blood flow (right). Scale bar, $100 \mu \mathrm{m}$ (left), $10 \mathrm{~ms}$, and $10 \mu \mathrm{m}$ (right) $(p=0.0747, t$-test, $n=64 \mathrm{BVs} / 4 \mathrm{mice}$ per group). (C) Quantification of the diameter of lymphatic ducts in mice treated with Cef or Veh in the SSS region ( $p=0.1316, t$-test, $n=5$ mice per group). (D) Quantification of the diameter of lymphatic ducts in mice treated with Cef or Veh in the TS region ( $p=0.1241, t$-test, $n=5$ mice per group). (E) Quantification of the diameter of BVs in mice treated with Cef or Veh in the SSS region ( $p=0.3436$, $t$-test, $n=5$ mice per group). (F) Sample images of A $\beta$ staining in the cortex of APP/PS1 mice treated with either Cef or Veh. Scale bar, $100 \mu \mathrm{m}$. (G) Quantification of amyloid plaque density in APP/PS1 mice treated with Cef or Veh ( $p=0.8199$, $t$-test, $n=4$ mice per group). (H) Sample images of Iba1 or GFAP staining in cortex (S1) of APP/PS1 mice treated with either Cef or Veh, for 5 days (left) or 15 days (right). Scale bar, 100 um. (I) Quantification of Iba1 density in APP/PS1 mice after treatment with Cef for either 5 days ( $p=0.0173, t$-test, $n=5$ mice per group) or 15 days $(p=0.0301, t$-test, $n=4$ mice per group). ( $\mathbf{J}$ ) Quantification of GFAP ${ }^{+}$area in APP/PS1 mice after treatment with Cef for either 5 days $(p=0.7982, t$-test, $n=5$ mice per group) or 15 days $(p=0.0447, t$-test, $n=4$ mice per group). Data are mean \pm SEM. ${ }^{*} p<0.05$; ${ }^{\star *} p<0.01$. 

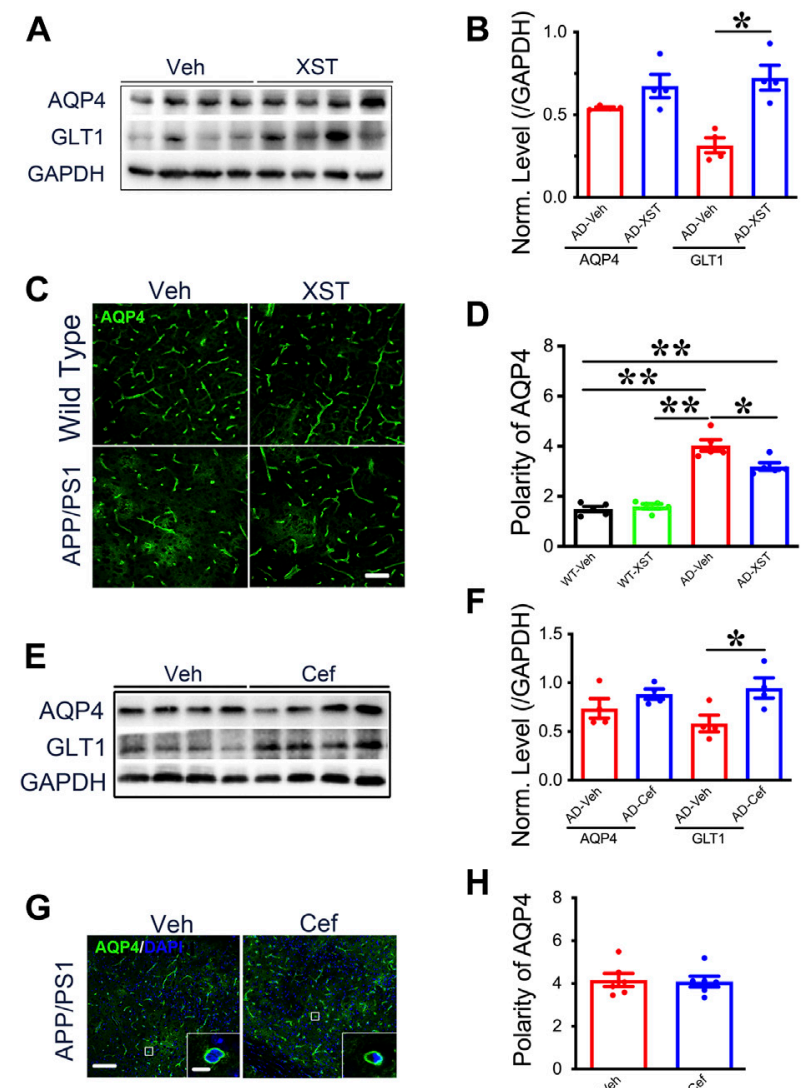

D

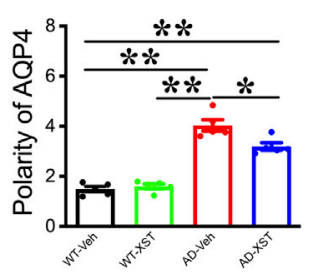

$\mathbf{F}$

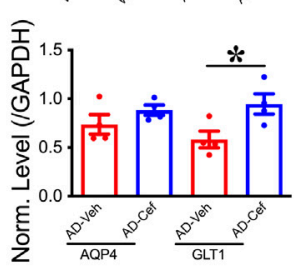

H

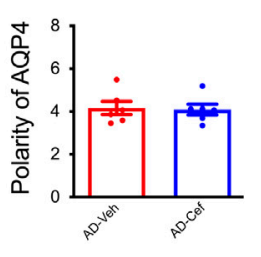

J
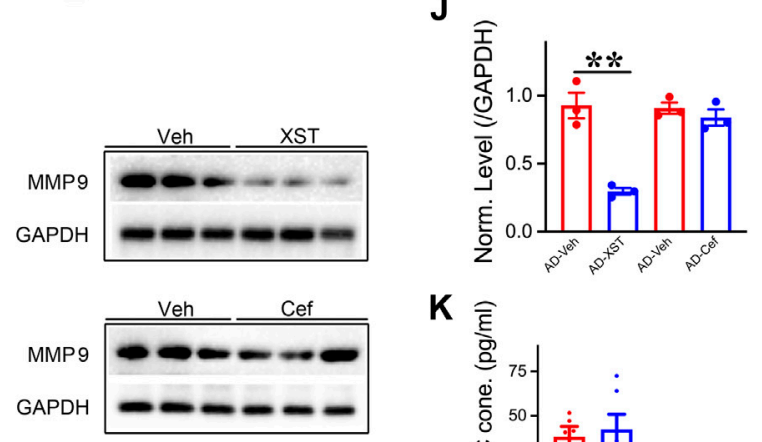

$\mathbf{K} \widehat{\bar{\varepsilon}}$

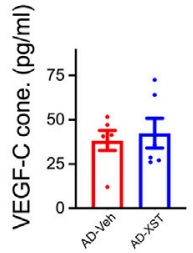

FIGURE 7 | Impacts of XST and Cef on the levels of GLT1, AQP4, and MMP-9 in APP/PS1 mice. (A) Sample images of WB analysis of brain AQP4 and GLT1 levels after treatment with XST in APP/PS1 mice. (B) Densitometric analysis of protein levels of AQP4 $(p=0.1113, t$-test, $n=4$ mice per group) and GLT1 ( $p=0.0035, t$-test, $n=4$ mice per group) after treatment with XST, normalized against GAPDH. (C) Sample images of AQP4 staining in cortex (S1) of APP/PS1 mice after treatment with XST for 15 days. Scale bar, $50 \mu \mathrm{m}$. (D) Quantification of AQP4 polarity after XST treatment $\left(F_{(1,16)}=\right.$ 183.4, C57 vs. AD, $p<0.0001$; AD-Veh vs. AD-XST, $p=0.0073$, two-way ANOVA with Bonferroni's multiple comparisons, $n=5$ mice per group). (E) Sample images of WB analysis of brain AQP4 and GLT1 levels after treatment with Cef in APP/PS1 mice. (F) Densitometric analysis of AQP4 $(p=0.1113$, $t$-test, $n=4$ mice per group) and GLT1 ( $p=0.035, t$-test, $n=4$ mice per group) levels after treatment with Cef, normalized to GAPDH. (G) Sample images of AQP4 in the cortex (S1) after treatment with Cef for 15 days in APP/PS1 mice.

(Continued)

FIGURE 7 | Scale bar, 50 mm (low). Scale bar, $10 \mu \mathrm{m}$ (high). (H) Quantification of AQP4 polarity after treatment with Cef for 15 days $(p=0.855$, $t$-test, $n=4$ mice per group). (I) Sample Western blots of brain MMP-9 levels after treatment with either XST (upper) or Cef (lower) in APP/PS1 mice. (J) Normalized levels of MMP-9 after treatment with XST ( $p=0.003, t$-test, $n=3$ mice per group) or Cef ( $p=0.390, t$-test, $n=3$ mice per group). (K) Quantitative measurement of VEGF-C levels in serum in WT and APP/PS1 mice ( $p=0.6928, t$-test, $n=6$ mice per group). Data are mean \pm SEM. ${ }^{*} p<$ $0.05 ;{ }^{\star *} p<0.01$

could influence astrocytic calcium signaling (Yang et al., 2016) and potassium homeostasis and is associated with the removal of interstitial $A \beta$ and modulation of glutamate-mediated synaptic transmission (Lan et al., 2017; Rasmussen et al., 2018) and AQP4 deficiency may also impair learning and memory, in part, through glutamate transporter-1 (GLT-1) (Lan et al., 2016a; Lan et al., 2016b). In addition, GLT-1 reduction and AQP4 alteration were reported in APP/PS1 mice (Takahashi et al., 2015; Benveniste et al., 2019). We found that XST treatment led to a significantly higher GLT-1 level but not AQP4 level in APP/PS1 mice (Figures 7A,B; $p<0.05$ ). The polarity of AQP4 (see Methods for quantification) was significantly higher in APP/PS1 mice compared to WT mice (Figures 6C,D; $p<0.01$ ), which reflects dysfunction of astrocytes (Lan et al., 2017). AQP4 polarity is significantly lower in the XST-treated than in Vehtreated APP/PS1 mice (Figures 7C,D; $p<0.05$ ), a change that was absent in the WT mice. Similar to XST treatment, a significantly higher GLT-1 protein level but no change in AQP4 level was seen in APP/PS1 mice treated with Cef, compared to the Veh-treated group (Figures 7E,F, $p<0.05$ ). However, AQP4 polarity was unchanged in Cef-injected APP/PS1 mice (Figures 7G,H). Since matrix metalloprotein 9 (MMP-9) has been implicated in targeting AQP4 to astrocyte endfeet (Yan et al., 2016; Amtul et al., 2018), we examined the level of MMP-9 in XST- or Ceftreated APP/PS1 mice. A significantly lower level of MMP-9 was seen only in the XST-treated group (Figures 7I,J, $p<0.01$ ), suggesting reduced AQP4 polarity being mediated by reduced MMP-9 level. Furthermore, MMP-2/MMP-9 is known to regulate lymphangiogenesis (Du et al., 2017) and previous studies have found that VEGF significantly increases lymphangiogenesis (Wong et al., 2016). Moreover, hippocampus is an important brain area affected in AD. Thus, we also measured the protein levels of GLT1, AQP4, and MMP9 in the hippocampus and found changes similar to those at the whole-brain level (Supplementary Figures S2A,B). However, we found VEGF-C level not different between XST- and Veh-treated groups in the serum of APP/PS1 mice (Figure 7K), suggesting that VEGF-C-independent signaling pathway may underlie the XST-induced improvement in the lymphatic system.

\section{DISCUSSION}

Due to the unmet medical need in finding effective AD drugs, most studies aimed to identify new AD drugs by testing their efficacy in reducing pathology and improving functions in $\mathrm{AD}$ model animals. For effective drugs/compounds, elucidating the 
potential novel mechanism underlying their action is important for a better understanding of AD pathology and new therapies. Our previous study showed significant improvements in brain functions (spatial learning and memory, motor learning, and memory), enhanced CBF, and reduced amyloid plaque size and density in the AD transgenic mice after XST injections. Here, we focus on immune responses and potential changes in LVs associated with XST treatment. We found the following with XST injections: 1) enlarged LV structures; 2 ) increased clearance/ removal of $A \beta$ from LVs and lymph nodes and reduced amyloid plaque size and density in the brain (Huang et al., 2018; Mesquita et al., 2018); 3) higher level of anti-inflammatory cytokines (IL-1 $\beta$ and IL-10) and lower level of proinflammatory cytokine (IL-6) in both serum and brain; 4) an initial higher activation of microglia and astrocytes followed by reduced activation over the subsequent weeks to month. These results suggest a potential of XST to modulate the structure of the lymphatic system in a functionally meaningful setting, providing a better mechanistic understanding of the therapeutic impacts of XST on AD and a potential using small molecules to enhance the structure/function of LVs.

\section{Rescue of LV Deficits After XST Treatment}

Meningeal LVs have recently been recognized as an important player in the complex circulation and exchange of soluble contents between CSF and ISF (Elham et al., 2020), and CSFISF exchange is a removal mechanism for macromolecules from the brain parenchyma to CSF (Jessen et al., 2015; Plog and Nedergaard, 2018). CSF circulation via the glymphatic system and meningeal LVs has been linked to neurological disorders (Rasmussen et al., 2018), including AD. Da Mesquita et al. (2018b) discovered that meningeal LVs drain macromolecules from the CNS (CSF and ISF) into the CLNs in mice. Agingassociated decrease in paravascular recirculation of CSF and ISF is likely to be responsible for the accumulation of $A \beta$ in the brain parenchyma (Benveniste et al., 2019), and brain-CSF-dural LVs pathway is an alternative route of $A \beta$ recirculation from brain ISF into the CSF sink (Sun et al., 2018b), supported by the presence of $\mathrm{A} \beta$ in the CLNs of AD transgenic mice (Karaman et al., 2018). Thus, an impaired meningeal lymphatic drainage of CSF may affect $\mathrm{A} \beta$ clearance and exacerbate amyloid burden in $\mathrm{AD}(\mathrm{He}$ et al., 2017; Da Mesquita et al., 2018a). In addition, mice lacking meningeal LVs show dysfunction in their ability to clear parenchymal macromolecules (Aspelund et al., 2015; Ma et al., 2017). Our current findings of significant $A \beta$ deposition in the meningeal lymphatics of APP/PS1 mice and significantly lower $\mathrm{A} \beta$ level in CLNs and meningeal LVs in XST-treated APP/PS1 mice are consistent with these findings. Hence, XST may increase meningeal LVs to remove A $\beta$ from the CSF of APP/PS1 mice. Previous reports have shown that upregulation of dural lymphangiogenesis is associated with significantly reduced senile amyloid plaques in APP/PS1 mice (Karaman et al., 2018; Da Mesquita et al., 2018b), consistent with our finding, whereas we do not have direct evidence for enhanced functions of the LV system or direct evidence for removal of $A \beta$ which is most directly revealed by imaging removal of injected fluorescent $A \beta$ into the brain or real-time monitoring of $A \beta$ clearance before and after XST administration. Due to technical reasons, we were not able to do this reliably. However, both our previous and current results have shown significant reduction in $A \beta$ level or plaque size in the APP/PS1 mouse brains after XST treatment and in reducing levels of both fibrillar and surrounding soluble $\mathrm{A} \beta$ (Huang et al., 2018). Thus, we suggest that the improved clearance of $A \beta$ may be related to the improved structures of LVs. In light of the ongoing debate on the functional contribution of LVs (Carare et al., 2020), we need to interpret our results with caution and need more direct examination to confirm the above hypothesis.

The newly discovered meningeal LVs are a novel path for cerebrospinal fluid drainage and represent a more conventional path for immune cells to egress the central nervous system (Louveau et al., 2015; Thomas et al., 2019). We found a significantly higher density of $\mathrm{T}$-cells $\left(\mathrm{CD} 3 \mathrm{e}^{+}\right)$in meningeal LVs of XST-treated APP/PS1 mice. In addition, IL-10 level was significantly increased and IL-6 level significantly decreased in the XST-treated group, consistent with reducing inflammation by XST (Da Mesquita et al., 2018b; Wang et al., 2019). We found that XST reduces the inflammatory responses in the serum and brains of transgenic AD mice, and this effect may be the result of improvements in LVs. Due to the potential multitarget effect of XST, it is quite possible that the modulation of inflammation is at least partially mediated by targets other than LVs. Reduced activation of microglia by XST may also ameliorate the production of proinflammatory cytokines, nitric oxide, and reactive oxygen species caused by excessive activation of microglia (Dando et al., 2018). It remains to be tested whether the initial elevated immune responses are a trigger for reducing $A \beta$ level which results in subsequently reduced inflammation.

\section{How Does XST Improve the Lymphatic System?}

One possibility is that XST increases the proliferation or activity of endothelial cells lining up the LVs. An alternative possibility is the improved LVs as an indirect effect of enhanced CBF. Many signal pathways are known to proliferate lymphatic endothelial cells and improve the lymphatic system function, including growth factors (VEGF, FGF, IGF, etc.) (Ren et al., 2017), Notch1/Akt (Blesinger et al., 2018; Sun et al., 2018a), MAPKERK1/2, and Wnt/ $\beta$-catenin pathway (Zhao et al., 2017; Cong et al., 2018). However, we did not find the VEGF-C level altered by XST treatment. One major in vivo effect of XST is elevated CBF in both AD and WT mice (Huang et al., 2018), and this efficacy has been the main rationale for XST's medical use in China (Du et al., 2015; Huang et al., 2018). Interestingly, Cef injection did not modulate LV structure and function albeit it significantly enhances CBF (Holschneider et al., 2020). Hence, despite the numerous and complex interactions between $\mathrm{CBF}$ and LVs (Kisler et al., 2017), directly enhancing CBF may not affect LVs, suggesting segregation of these two important fluid exchange systems in the brain. This is important not only for understanding the neurodegenerative diseases but also for development of successful therapeutic drugs. In addition, 
although both XST and Cef treatment lead to reduced inflammation in $\mathrm{AD}$ mice after 15 days, their paths are different: XST results in an initial activation of microglia followed by a decrease while Cef only leads to a significant decrease. Interestingly, XST results in a significant reduction in amyloid plaque level while Cef does not (Zumkehr et al., 2015; Fan et al., 2018).

Matrix metalloproteinase-9 (MMP-9) has been implicated in molecular mechanisms of AD (Kaminari et al., 2018), and MMP-9 expression plays an important role in excessive $A \beta$ deposition (Ning et al., 2014). In addition, MMP-2/-9mediated dystroglycan cleavage in astrocytes is likely to be crucial for maintaining the polarization of AQP4 (Yan et al., 2016; Zhang et al., 2018). The high expression of MMP-9 in the APP/PS1 mice (Kaminari et al., 2018; García-González et al., 2019) likely drives AQP4 to accumulate around amyloid plaques, hence showing high polarity. Glymphatic clearance mechanisms were strongly associated with AQP4 (Yin et al., 2018), and deletion of AQP4 in AD transgenic mice resulted in increased $A \beta$ burden and exacerbated cognitive impairment (Rasmussen et al., 2018). Thus, reduced MMP expression by XST may contribute to the low polarity of AQP4, while Cef (which does not affect MMP-9 level) does not have a similar effect. GLT-1 (EAAT2 in human), the dominant glutamate transporter in the cerebral cortex and hippocampus, is significantly reduced in $\mathrm{AD}$ patients and mice (Pereira et al., 2017; Sharma et al., 2019), and increased GLT-1 level by Cef treatment was associated with improved cognitive ability in APP/PS1 mice (Fan et al., 2018). Thus, it is possible that the observed improvement in cognitive functions in XST- or Ceftreated $\mathrm{AD}$ mice is at least partially mediated by increased GLT1 level.

\section{XST as Potential AD Drug by Improving Functions of LVs}

Here, we provided substantial evidence for a widely used Chinese herbal medicine with the potential of improving LVs in $\mathrm{AD}$ model mice. Although significant changes have been observed on XST's impact on BVs and LVs compared to the Veh-treated group, most of the improvements fell short of reversing to the level in the control/WT mice. This could be due to the following: 1) The treatment dose and duration which are not optimal. We have only tested one dose of XST which has been shown in improving cognitive functions and ameliorated $\mathrm{AD}$ pathology in APP/PS1 mice (Huang et al., 2018). It is possible that longer treatment may lead to better improvement. 2) The age of the AD mice. We have used $\mathrm{AD}$ mice at 11 months old which have developed prominent pathology and impaired functions. Although these mice are more likely to resemble $\mathrm{AD}$ patients when they receive medical treatment, the room for improvement is likely to be small. Future studies may test whether dosing XST in younger $\mathrm{AD}$ mice or with less advanced $\mathrm{AD}$ pathology may result in larger improvement. 3) Limited efficacy of XST as a drug on LV functions. Like other natural products, XST is not expected to exhibit high potency without significant chemical modification on a given biological function, and identification of key components in XST for improved efficacy and testing potential therapeutic path forward may be of interest.

\section{CONCLUSION}

In summary, the interesting finding of the current study is that XST effectively increases the size of LVs and improves A $\beta$ clearance ability of lymphatic systems. There is a potential to further develop for XST aiming to treat AD via enhancing LVs. Modulation of immune cells in the LVs and inflammatory cytokine levels via small molecules that can improve meningeal lymphatic functions might represent a novel and underexplored mechanism to improve brain functions, especially in the context of aging and neurodegeneration.

\section{DATA AVAILABILITY STATEMENT}

The original contributions presented in the study are included in the article/Supplementary Material; further inquiries can be directed to the corresponding author.

\section{ETHICS STATEMENT}

The animal study was reviewed and approved by all animal experiments and procedures were approved by the Ethics Committee of Peking University (Permit Number: AP0011).

\section{AUTHOR CONTRIBUTIONS}

$\mathrm{RZ}$ and $\mathrm{QZ}$ designed the study, $\mathrm{RZ}$ and $\mathrm{Y}-\mathrm{MH}$ conducted the experiments and analyzed data; $\mathrm{RZ}$ wrote the first draft of the paper; QZ edited the paper.

\section{FUNDING}

This work is supported by grants from Peking University Shenzhen Graduate School and Shenzhen Science and Technology Innovation Commission (2019SHIBS0004, SZBL2019062801003, and KQTD2015032709315529).

\section{ACKNOWLEDGMENTS}

We thank all the members in the Zhou Laboratories for critical discussions and Siyu Liu for help with ELISA.

\section{SUPPLEMENTARY MATERIAL}

The Supplementary Material for this article can be found online at: https://www.frontiersin.org/articles/10.3389/fphar.2021.605814/ full\#supplementary-material 


\section{REFERENCES}

Ahn, K. C., Learman, C. R., Dunbar, G. L., Maiti, P., Jang, W. C., Cha, H. C., et al. (2018). Characterization of Impaired Cerebrovascular Structure in APP/PS1 Mouse Brains. Neuroscience 385, 246-254. doi:10.1016/ j.neuroscience.2018.05.002

Amtul, Z., Yang, J., Nikolova, S., Lee, T. Y., Bartha, R., and Cechetto, D. F. (2018). The Dynamics of Impaired Blood-Brain Barrier Restoration in a Rat Model of Co-morbid Injury. Mol. Neurobiol. 55 (10), 8071-8083. doi:10.1007/s12035018-0904-4

Aspelund, A., Antila, S., Proulx, S. T., Karlsen, T. V., Karaman, S., Detmar, M., et al. (2015). A Dural Lymphatic Vascular System that Drains Brain Interstitial Fluid and Macromolecules. J. Exp. Med. 212 (7), 991-999. doi:10.1084/jem.20142290

Aspelund, A., Robciuc, M. R., Karaman, S., Makinen, T., and Alitalo, K. (2016). Lymphatic System in Cardiovascular Medicine. Circ. Res. 118 (3), 515-530. doi:10.1161/circresaha.115.306544

Bálint, L., Ocskay, Z., Deák, B. A., Aradi, P., and Jakus, Z. (2019). Lymph Flow Induces the Postnatal Formation of Mature and Functional Meningeal Lymphatic Vessels. Front. Immunol. 10, 3043. doi:10.3389/fimmu.2019.03043

Benveniste, H., Liu, X., Koundal, S., Sanggaard, S., Lee, H., and Wardlaw, J. (2019). The Glymphatic System and Waste Clearance with Brain Aging: A Review. Gerontology 65 (2), 106-119. doi:10.1159/000490349

Blesinger, H., Kaulfuß, S., Aung, T., Schwoch, S., Prantl, L., Rößler, J., et al. (2018). PIK3CA Mutations Are Specifically Localized to Lymphatic Endothelial Cells of Lymphatic Malformations. PLoS One 13 (7), e0200343. doi:10.1371/ journal.pone.0200343

Bracko, O., Njiru, B. N., Swallow, M., Ali, M., Haft-Javaherian, M., and Schaffer, C. B. (2019). Increasing Cerebral Blood Flow Improves Cognition into Late Stages in Alzheimer's Disease Mice. J. Cereb. Blood Flow Metab. 40, 1441-1452. doi: $10.1177 / 0271678 \times 19873658$

Brouillard, P., Boon, L., and Vikkula, M. (2014). Genetics of Lymphatic Anomalies. J. Clin. Invest. 124 (3), 898-904. doi:10.1172/jci71614

Carare, R. O., Aldea, R., Agarwal, N., Bacskai, B. J., Bechman, I., Boche, D., et al. (2020). Clearance of Interstitial Fluid (ISF) and CSF (CLIC) Group-Part of Vascular Professional Interest Area (PIA): Cerebrovascular Disease and the Failure of Elimination of Amyloid- $\beta$ from the Brain and Retina with Age and Alzheimer's Disease-Opportunities for Therapy. Alzheimers Dement (Amst) 12 (1), e12053. doi:10.1002/dad2.12053

Chen, W., Huang, P., Zeng, H., Lin, J., Shi, Z., and Yao, X. (2020). Cocaine-induced Structural and Functional Impairments of the Glymphatic Pathway in Mice. Brain Behav. Immun. 88, 97-104. doi:10.1016/j.bbi.2020.04.057

Cong, L., Zhang, Y., Huang, H., Cao, J., and Fu, X. (2018). DFMG Reverses Proliferation and Migration of Vascular Smooth Muscle Cells Induced by Coculture with Injured Vascular Endothelial Cells via Suppression of the TLR4Mediated Signaling Pathway. Mol. Med. Rep. 17 (4), 5692-5699. doi:10.3892/ mmr.2018.8635

Da Mesquita, S., Fu, Z., and Kipnis, J. (2018a). The Meningeal Lymphatic System: A New Player in Neurophysiology. Neuron 100 (2), 375-388. doi:10.1016/ j.neuron.2018.09.022

Da Mesquita, S., Louveau, A., Vaccari, A., Smirnov, I., Cornelison, R. C., Kingsmore, K. M., et al. (2018b). Functional Aspects of Meningeal Lymphatics in Ageing and Alzheimer's Disease. Nature 560 (7717), 185-191. doi:10.1038/s41586-018-0368-8

Da Mesquita, S., Louveau, A., Vaccari, A., Smirnov, I., Cornelison, R. C., Kingsmore, K. M., et al. (2018). Functional Aspects of Meningeal Lymphatics in Ageing and Alzheimer's Disease. Nature 560 (15), 185-191. doi:10.1038/s41586-018-0368-8

Dando, S. J., Kazanis, R., Chinnery, H. R., and McMenamin, P. G. (2018). Regional and Functional Heterogeneity of Antigen Presenting Cells in the Mouse Brain and Meninges. Glia 67 (8), 935-949. doi:10.1002/glia.23581

Dave, R. S., Jain, P., and Byrareddy, S. N. (2018). Functional Meningeal Lymphatics and Cerebrospinal Fluid Outflow. J. Neuroimmune Pharmacol. 13 (2), 123-125. doi:10.1007/s11481-018-9778-5

Du, H. T., Du, L. L., Tang, X. L., Ge, H. Y., and Liu, P. (2017). Blockade of MMP-2 and MMP-9 Inhibits Corneal Lymphangiogenesis. Graefes Arch. Clin. Exp. Ophthalmol. 255 (8), 1573-1579. doi:10.1007/s00417-017-3651-8
Du, R., Teng, J. F., Wang, Y., Zhao, X. Y., and Shi, Z. B. (2015). Clinical Study of Butylphthalide Combined with Xue Shuan Tong on Serum Inflammatory Factors and Prognosis Effect of Patients with Cerebral Infarction. Pak J. Pharm. Sci. 28 (5 Suppl. 1), 1823-1827.

Elham, E., Wumaier, R., Wang, C., Luo, X., Chen, T., and Zhong, N. (2020). Anatomic Evidence Shows that Lymphatic Drainage Exists in the Pituitary to Loop the Cerebral Lymphatic Circulation. Med. Hypotheses 143, 109898. doi:10.1016/j.mehy.2020.109898

Fakhoury, M. (2018). Microglia and Astrocytes in Alzheimer's Disease: Implications for Therapy. Curr. Neuropharmacol 16 (5), 508-518. doi:10.2174/1570159x15666170720095240

Fan, S., Xian, X., Li, L., Yao, X., Hu, Y., Zhang, M., et al. (2018). Ceftriaxone Improves Cognitive Function and Upregulates GLT-1-Related GlutamateGlutamine Cycle in APP/PS1 Mice. J. Alzheimers Dis. 66 (4), 1731-1743. doi:10.3233/jad-180708

Gao, X., Zheng, R., Ma, X., Gong, Z., Xia, D., and Zhou, Q. (2019). Elevated Level of PKM $\zeta$ Underlies the Excessive Anxiety in an Autism Model. Front. Mol. Neurosci. 12, 291. doi:10.3389/fnmol.2019.00291

García-González, L., Pilat, D., Baranger, K., and Rivera, S. (2019). Emerging Alternative Proteinases in APP Metabolism and Alzheimer's Disease Pathogenesis: A Focus on MT1-MMP and MT5-MMP. Front. Aging Neurosci. 11, 244. doi:10.3389/fnagi.2019.00244

Guimarães-Souza, E. M., Joselevitch, C., Britto, L. R. G., and Chiavegatto, S. (2019). Retinal Alterations in a Pre-clinical Model of an Autism Spectrum Disorder. Mol. Autism 10, 19. doi:10.1186/s13229-019-0270-8

Guo, H., Adah, D., James, P. B., Liu, Q., Li, G., Ahmadu, P., et al. (2018). Xueshuantong Injection (Lyophilized) Attenuates Cerebral Ischemia/ Reperfusion Injury by the Activation of Nrf2-VEGF Pathway. Neurochem. Res. 43 (5), 1096-1103. doi:10.1007/s11064-018-2523-x

He, Z. F., Chen, J., Zhou, C. N., Rao, Z., and Wang, X. H. (2017). Disabling Tremor Induced by Long-Term Use of Sodium Valproate and Lamotrigine: Case Report. Medicine (Baltimore) $96 \quad$ (47), e8711. doi:10.1097/ MD.0000000000008711

Holschneider, D. P., Wang, Z., Chang, H., Zhang, R., Gao, Y., Guo, Y., et al. (2020). Ceftriaxone Inhibits Stress-induced Bladder Hyperalgesia and Alters Cerebral Micturition and Nociceptive Circuits in the Rat: A Multidisciplinary Approach to the Study of Urologic Chronic Pelvic Pain Syndrome Research Network Study. Neurourology and Urodynamics 39, 1628-1643. doi:10.1002/nau.24424

Huang, Y., Guo, B., Shi, B., Gao, Q., and Zhou, Q. (2018). Chinese Herbal Medicine Xueshuantong Enhances Cerebral Blood Flow and Improves Neural Functions in Alzheimer's Disease Mice. J. Alzheimers Dis. 63 (3), 1089-1107. doi:10.3233/ jad-170763

Jessen, N. A., Munk, A. S., Lundgaard, I., and Nedergaard, M. (2015). The Glymphatic System: A Beginner's Guide. Neurochem. Res. 40 (12), 2583-2599. doi:10.1007/s11064-015-1581-6

Kametani, F., and Hasegawa, M. (2018). Reconsideration of Amyloid Hypothesis and Tau Hypothesis in Alzheimer's Disease. Front. Neurosci. 12, 25 doi:10.3389/fnins.2018.00025

Kaminari, A., Tsilibary, E. C., and Tzinia, A. (2018). A New Perspective in Utilizing MMP-9 as a Therapeutic Target for Alzheimer's Disease and Type 2 Diabetes Mellitus. J. Alzheimers Dis. 64 (1), 1-16. doi:10.3233/jad-180035

Karaman, S., Leppänen, V. M., and Alitalo, K. (2018). Vascular Endothelial Growth Factor Signaling in Development and Disease. Development 145 (14). doi:10.1242/dev.151019

Kaur, D., Sharma, V., and Deshmukh, R. (2019). Activation of Microglia and Astrocytes: a Roadway to Neuroinflammation and Alzheimer's Disease. Inflammopharmacology 27 (4), 663-677. doi:10.1007/s10787-019-00580-x

Kim, K. W., and Song, J. H. (2017). Emerging Roles of Lymphatic Vasculature in Immunity. Immune Netw. 17 (1), 68-76. doi:10.4110/in.2017.17.1.68

Kisler, K., Nelson, A. R., Montagne, A., and Zlokovic, B. V. (2017). Cerebral Blood Flow Regulation and Neurovascular Dysfunction in Alzheimer Disease. Nat. Rev. Neurosci. 18 (7), 419-434. doi:10.1038/nrn.2017.48

Klotz, L., Norman, S., Vieira, J. M., Masters, M., Rohling, M., Dubé, K. N., et al. (2015). Cardiac Lymphatics Are Heterogeneous in Origin and Respond to Injury. Nature 522 (7554), 62-67. doi:10.1038/nature14483

Koronyo-Hamaoui, M., Ko, M. K., Koronyo, Y., Azoulay, D., Seksenyan, A., Kunis, G., et al. (2009). Attenuation of AD-like Neuropathology by Harnessing 
Peripheral Immune Cells: Local Elevation of IL-10 and MMP-9. J. Neurochem. 111 (6), 1409-1424.

Kortela, E., Hytönen, J., Numminen, J., Overmyer, M., Saxen, H., and Oksi, J. (2017). Cerebral Vasculitis and Intracranial Multiple Aneurysms in a Child with Lyme Neuroborreliosis. JMM Case Rep. 4 (4), e005090. doi:10.1099/ jmmcr.0.005090

Kyrtsos, C., and Baras, J. (2014). “The Glymphatic System and Alzheimer's Disease: Possible Connection? ”. The Sixth International Conference on Bioinformatics, Biocomputational Systems and Biotechnologies.

Lan, Y. L., Chen, J. J., Hu, G., Xu, J., Xiao, M., and Li, S. (2017). Aquaporin 4 in Astrocytes Is a Target for Therapy in Alzheimer's Disease. Curr. Pharm. Des. 23 (33), 4948-4957. doi:10.2174/1381612823666170714144844

Lan, Y. L., Zhao, J., Ma, T., and Li, S. (2016a). The Potential Roles of Aquaporin 4 in Alzheimer's Disease. Mol. Neurobiol. 53 (8), 5300-5309. doi:10.1007/s12035015-9446-1

Lan, Y. L., Zou, S., Chen, J. J., Zhao, J., and Li, S. (2016b). The Neuroprotective Effect of the Association of Aquaporin-4/Glutamate Transporter-1 against Alzheimer's Disease. Neural Plast. 2016, 4626593. doi:10.1155/2016/4626593

Li, R. L., Wang, J. X., Chai, L. J., Guo, H., Wang, H., Chen, L., et al. (2020). Xueshuantong for Injection (Lyophilized, ) Alleviates Streptozotocin-Induced Diabetic Retinopathy in Rats. Chin. J. Integr. Med. 26 (11), 825-832. doi:10.1007/s11655-020-3088-5

Louveau, A., Plog, B. A., Antila, S., Alitalo, K., Nedergaard, M., and Kipnis, J. (2017). Understanding the Functions and Relationships of the Glymphatic System and Meningeal Lymphatics. J. Clin. Invest. 127 (9), 3210-3219. doi:10.1172/jci90603

Louveau, A., Smirnov, I., Keyes, T. J., Eccles, J. D., Rouhani, S. J., Peske, J. D., et al. (2015). Structural and Functional Features of central Nervous System Lymphatic Vessels. Nature 523 (7560), 337-341. doi:10.1038/nature14432

Ma, Q., Ineichen, B. V., Detmar, M., and Proulx, S. T. (2017). Outflow of Cerebrospinal Fluid Is Predominantly through Lymphatic Vessels and Is Reduced in Aged Mice. Nat. Commun. 8 (1), 1434. doi:10.1038/s41467-01701484-6

Ning, M., Wu, Y., Ni, X., Zhao, Y., and Ling, R. (2014). Cerebral Microemboli Increase $\beta$-amyloid Protein Deposition, MMP-9, and GFAP Expression in the Alzheimer's Model of APP/PS1 Double Transgenic Mice. Curr. Neurovasc Res. 11 (3), 190-201. doi:10.2174/1567202611666140606104132

Patel, A., Simon, S., M Elangoven, I., Amalchandran, J., and S Jain, T. (2019). Dopamine Transporter Maging with Tc-99m TRODAT-1 SPECT in Parkinson's Isease and its Orrelation with Linical Isease Everity. Asia Ocean J. Nucl. Med. Biol. 7 (1), 22-28. doi:10.22038/AOJNMB.2018.30356.1208

Pereira, A. C., Gray, J. D., Kogan, J. F., Davidson, R. L., Rubin, T. G., Okamoto, M., et al. (2017). Age and Alzheimer's Disease Gene Expression Profiles Reversed by the Glutamate Modulator Riluzole. Mol. Psychiatry 22 (2), 296-305. doi:10.1038/mp.2016.33

Plog, B. A., and Nedergaard, M. (2018). The Glymphatic System in Central Nervous System Health and Disease: Past, Present, and Future. Annu. Rev. Pathol. 13, 379-394. doi:10.1146/annurev-pathol-051217-111018

Polanco, J. C., Li, C., Bodea, L. G., Martinez-Marmol, R., Meunier, F. A., and Götz, J. (2018). Amyloid- $\beta$ and Tau Complexity - towards Improved Biomarkers and Targeted Therapies. Nat. Rev. Neurol. 14 (1), 22-39. doi:10.1038/ nrneurol.2017.162

Rasmussen, M. K., Mestre, H., and Nedergaard, M. (2018). The Glymphatic Pathway in Neurological Disorders. Lancet Neurol. 17 (11), 1016-1024. doi:10.1016/s1474-4422(18)30318-1

Ren, J. G., Xia, H. F., Yang, J. G., Zhu, J. Y., Zhang, W., Chen, G., et al. (2017). Down-regulation of Polycystin in Lymphatic Malformations: Possible Role in the Proliferation of Lymphatic Endothelial Cells. Hum. Pathol. 65, 231-238. doi:10.1016/j.humpath.2017.05.016

Schläger, C., Körner, H., Krueger, M., Vidoli, S., Haberl, M., Mielke, D., et al. (2016). Effector T-Cell Trafficking between the Leptomeninges and the Cerebrospinal Fluid. Nature 530 (7590), 349-353. doi:10.1038/ nature 16939

Sharma, A., Kazim, S. F., Larson, C. S., Ramakrishnan, A., Gray, J. D., McEwen, B. S., et al. (2019). Divergent Roles of Astrocytic versus Neuronal EAAT2 Deficiency on Cognition and Overlap with Aging and Alzheimer's Molecular Signatures. Proc. Natl. Acad. Sci. U S A. 116 (43), 21800-21811. doi:10.1073/pnas.1903566116
Shechter, R., London, A., and Schwartz, M. (2013). Orchestrated Leukocyte Recruitment to Immune-Privileged Sites: Absolute Barriers versus Educational gates. Nat. Rev. Immunol. 13 (3), 206-218. doi:10.1038/nri3391

Simone, L., Pisani, F., Mola, M. G., De Bellis, M., Merla, G., Micale, L., et al. (2019). AQP4 Aggregation State Is a Determinant for Glioma Cell Fate. Cancer Res. 79 (9), 2182-2194. doi:10.1158/0008-5472.Can-18-2015

Sun, B., Dong, C., Lei, H., Gong, Y., Li, M., Zhang, Y., et al. (2018a). Propranolol Inhibits Proliferation and Invasion of Hemangioma-Derived Endothelial Cells by Suppressing the DLL4/Notch1/Akt Pathway. Chem. Biol. Interact 294, 28-33. doi:10.1016/j.cbi.2018.08.018

Sun, B. L., Wang, L. H., Yang, T., Sun, J. Y., Mao, L. L., Yang, M. F., et al. (2018b). Lymphatic Drainage System of the Brain: A Novel Target for Intervention of Neurological Diseases. Prog. Neurobiol. 163-164, 118-143. doi:10.1016/ j.pneurobio.2017.08.007

Takahashi, K., Foster, J. B., and Lin, C. L. (2015). Glutamate Transporter EAAT2: Regulation, Function, and Potential as a Therapeutic Target for Neurological and Psychiatric Disease. Cell Mol Life Sci 72 (18), 3489-3506. doi:10.1007/ s00018-015-1937-8

Tarasoff-Conway, J. M., Carare, R. O., Osorio, R. S., Glodzik, L., Butler, T., Fieremans, E., et al. (2016). Clearance Systems in the Brain-Iimplications for Alzheimer Diseaser. Nat. Rev. Neurol. 12 (4), 248. doi:10.1038/ nrneurol.2016.36

Thomas, J. L., Jacob, L., and Boisserand, L. (2019). [Lymphatic System in central Nervous System]. Med. Sci. (Paris) 35 (1), 55-61. doi:10.1051/medsci/2018309

Toly-Ndour, C., Lui, G., Nunes, M. M., Bruley-Rosset, M., Aucouturier, P., and Dorothée, G. (2011). MHC-independent Genetic Factors Control the Magnitude of CD4+ $\mathrm{T}$ Cell Responses to Amyloid- $\beta$ Peptide in Mice through Regulatory $\mathrm{T}$ Cell-Mediated Inhibition. J. Immunol. 187 (9), 4492-4500. doi:10.4049/jimmunol.1003953

van der Kleij, L. A., Petersen, E. T., Siebner, H. R., Hendrikse, J., Frederiksen, K. S., Sobol, N. A., et al. (2018). The Effect of Physical Exercise on Cerebral Blood Flow in Alzheimer's Disease. Neuroimage Clin. 20, 650-654. doi:10.1016/ j.nicl.2018.09.003

Venero Galanternik, M., Stratman, A. N., Jung, H. M., Butler, M. G., and Weinstein, B. M. (2016). Building the Drains: The Lymphatic Vasculature in Health and Disease. Wiley Interdiscip. Rev. Dev. Biol. 5 (6). doi:10.1002/ wdev. 246

Vogelsang, P., Giil, L. M., Lund, A., Vedeler, C. A., Parkar, A. P., Nordrehaug, J. E., et al. (2018). Reduced Glucose Transporter-1 in Brain Derived Circulating Endothelial Cells in Mild Alzheimer's Disease Patients. Brain Res. 1678, 304-309. doi:10.1016/j.brainres.2017.10.035

Wang, F. J., Wang, S. X., Chai, L. J., Zhang, Y., Guo, H., and Hu, L. M. (2018). Xueshuantong Injection (Lyophilized) Combined with Salvianolate Lyophilized Injection Protects against Focal Cerebral Ischemia/reperfusion Injury in Rats through Attenuation of Oxidative Stress. Acta Pharmacol. Sin 39 (6), 998-1011. doi:10.1038/aps.2017.128

Wang, L., Zhang, Y., Zhao, Y., Marshall, C., Wu, T., and Xiao, M. (2019). Deep Cervical Lymph Node Ligation Aggravates AD-like Pathology of APP/PS1 Mice. Brain Pathol. 29 (2), 176-192. doi:10.1111/bpa.12656

Wang, M., Iliff, J. J., Liao, Y., Chen, M. J., Shinseki, M. S., Venkataraman, A., et al. (2012). Cognitive Deficits and Delayed Neuronal Loss in a Mouse Model of Multiple Microinfarcts. J. Neurosci. 32 (50), 17948-17960. doi:10.1523/ jneurosci.1860-12.2012

Wang, X., Wang, S., Wang, J., Guo, H., Dong, Z., Chai, L., et al. (2015). Neuroprotective Effect of Xueshuantong for Injection (Lyophilized) in Transient and Permanent Rat Cerebral Ischemia Model. Evid. Based Complement. Alternat Med. 2015, 134685. doi:10.1155/2015/134685

Williams, A. S., Jones, S. G., Goodfellow, R. M., Amos, N., and Williams, B. D. (1999). Interleukin-1beta (IL-1beta) Inhibition: a Possible Mechanism for the Anti-inflammatory Potency of Liposomally Conjugated Methotrexate Formulations in Arthritis. Br. J. Pharmacol. 128 (1), 234-240. doi:10.1038/ sj.bjp. 0702776

Wong, H. L., Jin, G., Cao, R., Zhang, S., Cao, Y., and Zhou, Z. (2016). MT1-MMP Sheds LYVE-1 on Lymphatic Endothelial Cells and Suppresses VEGF-C Production to Inhibit Lymphangiogenesis. Nat. Commun. 7, 10824. doi:10.1038/ncomms10824

Yan, W., Zhao, X., Chen, H., Zhong, D., Jin, J., Qin, Q., et al. (2016). $\beta$-Dystroglycan Cleavage by Matrix Metalloproteinase-2/-9 Disturbs Aquaporin-4 Polarization 
and Influences Brain Edema in Acute Cerebral Ischemia. Neuroscience 326, 141-157. doi:10.1016/j.neuroscience.2016.03.055

Yang, C., Huang, X., Huang, X., Mai, H., Li, J., Jiang, T., et al. (2016). Aquaporin-4 and Alzheimer's Disease. J. Alzheimers Dis. 52 (2), 391-402. doi:10.3233/jad-150949

Yao, C., Yang, W., Zhang, J., Qiu, S., Chen, M., Shi, X., et al. (2017). UHPLC-QTOF-MS-based Metabolomics Approach to Compare the Saponin Compositions of Xueshuantong Injection and Xuesaitong Injection. J. Sep. Sci. 40 (4), 834-841. doi:10.1002/jssc.201601122

Yin, M., Pu, T., Wang, L., Marshall, C., Wu, T., and Xiao, M. (2018). Astroglial Water Channel Aquaporin 4-mediated Glymphatic Clearance Function: A Determined Factor for Time-Sensitive Treatment of Aerobic Exercise in Patients with Alzheimer's Disease. Med. Hypotheses 119, 18-21. doi:10.1016/ j.mehy.2018.07.016

Zhang, H. Y., Niu, W., Olaleye, O. E., Du, F. F., Wang, F. Q., Huang, Y. H., et al. (2020). Comparison of Intramuscular and Intravenous Pharmacokinetics of Ginsenosides in Humans after Dosing XueShuanTong, a Lyophilized Extract of Panax Notoginseng Roots. J. Ethnopharmacol 253, 112658. doi:10.1016/ j.jep.2020.112658

Zhang, Z., Zhang, L., Chen, J., Cao, Y., Qu, M., Lin, X., et al. (2018). 2-(2-Benzofuranyl)2-Imidazoline Mediates Neuroprotection by Regulating the Neurovascular Unit Integrity in a Rat Model of Focal Cerebral Ischemia. J. Stroke Cerebrovasc. Dis. 27 (6), 1481-1489. doi:10.1016/j.jstrokecerebrovasdis.2017.12.041

Zhao, B., Zhao, Z., Sun, X., Zhang, Y., Guo, Y., Tian, P., et al. (2017). Effect of Micro Strain Stress on Proliferation of Endothelial Progenitor Cells In Vitro by the MAPK-Erk1/2 Signaling Pathway. Biochem. Biophys. Res. Commun. 492 (2), 206-211. doi:10.1016/j.bbrc.2017.08.050
Zhu, T., Zhang, F., Li, H., He, Y., Zhang, G., Huang, N., et al. (2019). Long-term Icariin Treatment Ameliorates Cognitive Deficits via CD4+ T Cell-Mediated Immuno-Inflammatory Responses in APP/PS1 Mice. Clin. Interv. Aging 14, 817-826. doi:10.2147/cia.S208068

Zumkehr, J., Rodriguez-Ortiz, C. J., Cheng, D., Kieu, Z., Wai, T., Hawkins, C., et al. (2015). Ceftriaxone Ameliorates Tau Pathology and Cognitive Decline via Restoration of Glial Glutamate Transporter in a Mouse Model of Alzheimer's Disease. Neurobiol. Aging 36 (7), 2260-2271. doi:10.1016/ j.neurobiolaging.2015.04.005

Conflict of Interest: The authors declare that the research was conducted in the absence of any commercial or financial relationships that could be construed as a potential conflict of interest.

Publisher's Note: All claims expressed in this article are solely those of the authors and do not necessarily represent those of their affiliated organizations or those of the publisher, the editors, and the reviewers. Any product that may be evaluated in this article or claim that may be made by its manufacturer is not guaranteed or endorsed by the publisher.

Copyright $\odot 2021$ Zheng, Huang and Zhou. This is an open-access article distributed under the terms of the Creative Commons Attribution License (CC BY). The use, distribution or reproduction in other forums is permitted, provided the original author(s) and the copyright owner(s) are credited and that the original publication in this journal is cited, in accordance with accepted academic practice. No use, distribution or reproduction is permitted which does not comply with these terms. 